\title{
Topographically distinct cortical activation in episodic long-term memory: The retrieval of spatial versus verbal information
}

\author{
MARTIN HEIL, FRANK RÖSLER, and ERWIN HENNIGHAUSEN \\ Philipps-University, Marburg, Germany
}

\begin{abstract}
Two experiments are reported to study slow potentials in the EEG during reactivation of spatial and verbal information. Subjects had to learn associations between drawings and one, two, or three mediators (locations in Experiment 1, nouns in Experiment 2). During recall, subjects had to decide whether or not two drawings were linked to each other by a common mediator. EEG was recorded during learning and recall. Both experiments were completely equivalent. Irrespective of the quality of the mediators, response time proved to be a linear function of the numbers of mediators to be recalled. Negative slow potentials that accompanied the reactivation of information during anticipation learning and cued recall had a material-specific topography: The maximum was found over the parietal cortex for spatial information and over the left frontal cortex for verbal information. Moreover, the amplitude at these scalp locations varied with the amount of the to-be-retrieved information. The results support the claim of topographically distinct cell assemblies specialized for storage and retrieval of distinct kinds of information.
\end{abstract}

A major controversy in cognitive psychology concerns the question of whether memory contents are stored in one common code only or in several distinct, material-specific codes (e.g., Anderson, 1976; Kosslyn, 1994; Paivio, 1986). Empirical tests of this controversy rest on the assumption that the dynamics of memory activation depend on the type of representation to be accessed. As a consequence, response time (RT) functions or error rates are studied for systematic processing differences in settings in which distinct codes (e.g., verbal and nonverbal contents) have to be reactivated. Although a great number of ingeniously planned experiments were run with this approach, the question of one common code versus several distinct codes could not yet be settled satisfactorily. First, there are general epistemological problems that restrict the possible conclusions to be drawn from RT data about codes and processes (Anderson, 1978). Second, the empirical evidence itself is equivocal. Some findings support the position of a separate code (e.g., Stopher \& Kirsner, 1981), whereas others do not (e.g., Anderson \& Paulson, 1978).

More recent approaches of cognitive neuroscience deal with the same question, but from a different perspective.

This study was supported by the German Research Foundation (DFG) Grant Ro 529 assigned to F.R. The authors gratefully acknowledge the helpful discussions with N. Cowan, S. M. Kosslyn, H. Lachnit, R. Mangun, H. Scheiblechner, and G. Stemmler. The authors also thank K. T. Spoehr, L. Nadel, and an anonymous reviewer for helpful comments on an earlier version of this article, and J. Bajrić, A. Löll, and $S$. Rupp for their support during data acquisition. The authors are grateful to J. Bajric for the preparation of the figures. Correspondence should be addressed to M. Heil, Department of Psychology, Philipps-University, Gutenbergstrasse 18, D-35032 Marburg, Germany (e-mail: heil@mailer. uni-marburg.de).
Referring to brain lesion or brain imagery data, respectively, the question is whether there exist(s) either only one common, multipurpose storage system or, instead, several distinct systems that are specialized for the storage of material-specific information (e.g., Frackowiak, 1994; Nadel, 1992; Squire, 1992). Another question is, where in the brain may this memory system(s) be localized? One alternative is that the representations are stored in the same cell assemblies in which the on-line processing of information is performed during perception and action (i.e., in the sensory and motor projection areas and in multisensory integration areas; Damasio, 1989). Alternatively, besides those specialized for on-line processing, cell assemblies might exist that are specialized only for storage and retrieval of all types of information (Squire, 1992).

From a more theoretical perspective, Hinton, McClelland, and Rumelhart (1986) came to the conclusion that similarly organized, but material-specific, neural networks localized within distinct parts of the human brain may be the most likely basis for storage and retrieval. They argue that

\section{a system that uses distributed representations still requires many different modules for representing completely dif- ferent things.... For example, different modules would be devoted to things as different as mental images and sentence structure, but two different mental images would correspond to alternative patterns of activity in the same module. The representations advocated here are local at a global scale but global at a local scale. (Hinton et al., 1986, p. 79)}

Under these assumptions, memory retrieval means that a cue triggers a pattern of neuronal activity in those cortical modules in which the respective information was also processed originally (see also Damasio, 1989; von der 
Malsburg, 1981). This perspective of multimodal storage systems could be seen as the neurophysiological version of cognitive theories, which postulate material-specific codes for storage and retrieval.

Considering the macro- and microanatomy of the central nervous system, such a theoretical framework has a great appeal. Studies of the cytoarchitectonics of the brain show that the neocortex is structured into distinct modules and that each module consists of a large set of network-like cell assemblies. These assemblies are very likely specialized for both processing and storage of information (e.g., Braitenberg \& Schütz, 1991). Thus, the hypothesis of cognitive neuroscientists about the architecture of human memory is in good agreement with anatomical findings. Also, behavioral effects observed in brain lesioned patients fit into this model (e.g., De Renzi, 1982; McCarthy \& Warrington, 1990, ch. 15). However, all this evidence is post hoc; what is missing is some direct empirical evidence for the prediction that distinct cortical activation patterns are evoked during retrieval of different types of representations.

Taking the theoretical framework of Hinton et al. (1986) as a starting point, specific hypotheses about the topography of cortical activation during memory retrieval can be formulated. On the basis of lesion and other physiological data, it can be predicted that the retrieval of spatial information should be accompanied by an activation of the parietal cortex, whereas the retrieval of verbal in formation should result in an activation of the left frontal cortex. Spatial processing as a function of the parietal cortex was demonstrated, for example, by single cell recordings (Georgopoulos, Lurito, Petrides, Schwartz, \& Massey, 1989) and experimentally induced lesions in monkeys (Mishkin, Ungerleider, \& Macko, 1983), by the study of neuropsychological symptoms of patients with lesions (Levine, Warach, \& Farah, 1985), and by brain imaging techniques (positron-emission tomography, PET) applied to healthy human subjects in experimental settings (Horwitz et al., 1992; Köhler, Kapur, Moscovitsch, \& Houle, 1995). The importance of the left frontal cortex for verbal memory processes has been demonstrated by converging evidence from the analyses of lesion effects (Jetter, Poser, Freeman, \& Markowitsch, 1986; Risse, Rubens, \& Jordan, 1984) and by brain imaging data (Peterson, Fox, Posner, Mintun, \& Raichle, 1988).

In the present paper, two experiments are reported that tested explicitly whether distinct cortical cell assemblies are activated, if verbal and spatial contents have to be accessed, and whether these activity changes are specific manifestations of memory retrieval. In a previous study (Rösler, Heil, \& Hennighausen, 1995), we were able to show that distinct cortical activation patterns can be evoked, if verbal, spatial, and color associations, respectively, are reactivated in a controlled retrieval experiment. In the two experiments reported here, these findings were extended in two respects. First, the results show that the activity level of a code-specific cell assembly increases if more representations of a particular code have to be accessed. This amplitude change is topographically restricted to assemblies that are specific for a particular code. Cell assemblies that are specific for other codes do not show the effect. Second, the results reveal that the activity changes are independent from the type of retrieval process. The very same cell assemblies are activated if representations of a particular code are accessed either during anticipation learning or during cued recall taking place 1 day after the learning session. These data clearly support cognitive theories that postulate distinct memory codes, and they substantiate the idea that stored representations are reactivated in code-specific cell assemblies that are localized in restricted cortical areas. Before going into the details of the two experiments, we will briefly summarize the logic and the advantages of our experimental paradigm, and we will explain why particular measures of brain activity - the slow event-related brain potentials (ERPs) of the electroencephalography (EEG)are most suitable to monitor activity changes of cortical cell assemblies during memory retrieval.

\section{The Modified Recall Paradigm to Study Genuine Memory Retrieval}

For investigating cortical activation patterns during memory retrieval, a variant of the fan paradigm (Anderson, 1974) was used. The fan effect refers to the finding that subjects are slower to recognize particular information about a fact, the more the related information they have learned about this fact before. Usually, to study the fan effect, subjects are first trained with episodic associations having an experimentally controlled fan of different size. Later, their recognition memory is tested with targets and distractors (i.e., with learned and other previously unlearned items). Unfortunately, this standard fan paradigm has the same disadvantages as do all recognition memory tests in which learned targets and unlearned distractors are combined. In such a situation, performance may depend not only on retrieval processes but also on plausibility or consistency judgments about whether an item is old or new (for a detailed discussion of these problems, see Heil, Rösler, \& Hennighausen, 1994). To circumvent this problem, we developed a modified recall paradigm (Heil et al., 1994). The design enforces genuine memory recall whenever a test item is presented, because other strategies would be useless to solve the task. Moreover, as it will become evident from the following paragraphs, the design can easily be adapted to test the reactivation of different memory codes or of different amounts of information within one code domain.

In this modified fan paradigm, subjects have to learn associations between target items and "mediators." The targets are, for example, elaborated drawings that represent animate or inanimate objects of categories (e.g., leaves, fishes, or windmills). One, two, or three such exemplars (in our terminology, specific concepts) may belong to the same category. Thus, there are, for example, three different leaves, but only one fish (see Figure 1). 

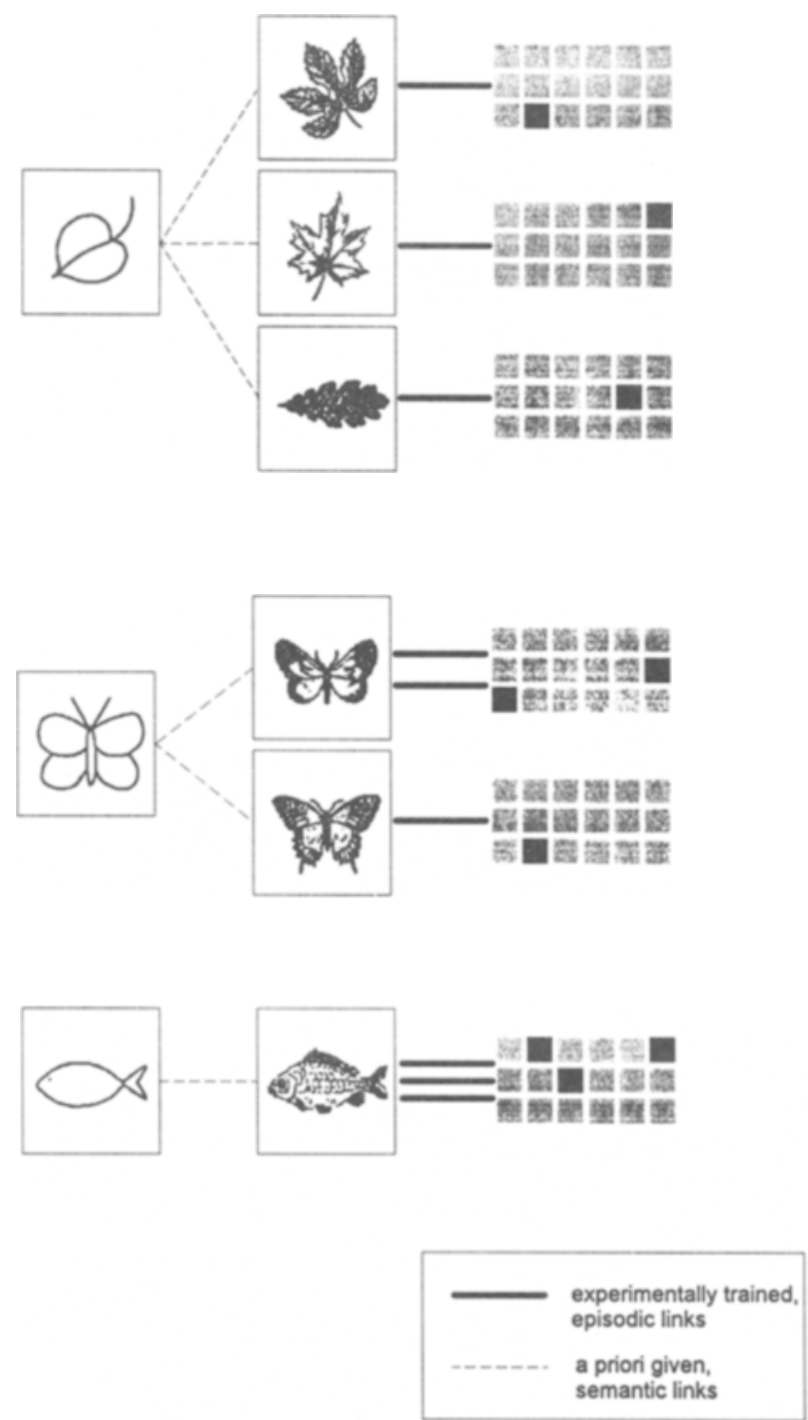

Figure 1. Examples of general concepts, specific concepts, and mediators, and the associative structure that was established in the modified recall paradigm. The subjects had to learn the associations between the elaborated drawings and the locations in the grid. The associations between the unelaborated drawings and the elaborated ones were not experimentally trained but were a priori given and used in the retrieval test.

The fan of the specific concepts in relation to the mediators is varied systematically - that is, there are one, two, or three mediators associated with a given specific concept. Mediators can be realized by a variety of different items. In a "spatial" condition (our Experiment 1), positions in a grid of $6 \times 3$ gray squares, as shown in Figure 1 , are used as mediators. In a verbal condition (our Experiment 2), mediators are defined by nouns denoting concrete objects. The associations between specific concepts and mediators are learned extensively until hardly any errors are committed in cued recall. In the test situation, RT is measured while subjects have to decide whether two drawings presented together side by side are linked to each other via one common mediator (i.e., whether both drawings combined in a probe are associ- ated with the same position). Thus, a memory probe does not refer, as in Anderson (1974), to an existing or a nonexisting direct associative link between two concepts. Instead, the associated mediators (the spatial positions or the concrete nouns) have to be reactivated in memory first before they can be checked for an existing episodic link. It is clear that both positive and negative probes necessarily invoke genuine memory recall. Plausibility judgments about whether presented information is old or new will not help to solve the task. Only old information is presented, and the task is to check for the existence or nonexistence of entries in episodic memory. This design was realized in Heil et al. (1994) with three different types of mediators: positions, nouns, and color patches. RT proved to be a linear function of the number of associated mediators (the fan) with virtually the same slope for all types of mediators.

The modified recall design provides another interesting feature for studying particular aspects of the retrieval process. In addition to the probes constructed from explicitly learned specific concepts (the elaborate drawings in Figure 1), probes can also be introduced, in the test session, that are constructed from "general concepts." These general concepts are unelaborated drawings depicting only the essential features of the semantic categories of the specific concepts (left column in Figure 1). These general concepts are not presented in the learning session, and, consequently, no episodic associations are established between the general concepts and the mediators or between the general and specific concepts. Links between the general and specific concepts exist only on the basis of a priori given semantic knowledge. When encountering a probe constructed from two general concepts, the subject had to decide whether there exists a mediator that is associated to the specific concepts subordinated to the general concepts. To solve this task, the subject has first to activate the semantically associated specific concepts (via the dashed lines in Figure 1), and, after this, he or she can activate the mediators (via the continuous lines in Figure 1). Depending on the fan of the specific concepts, a general concept "points" to one, two, or three specific concepts that are semantically subordinated (i.e., with respect to the specific concepts, the general concepts have a nominal fan of 1,2 , or 3 ). However, the sum of the mediators that are associated to all of the specific concepts of one semantic category (subordinate to one general concept) is constant (i.e., a general concept always has a fan of 3 with respect to the mediators [the grid positions]). In Heil et al.'s (1994) Experiments 4 and 5 , the slope of the RT function obtained with probes constructed from general concepts was approximately the same as with those constructed from specific concepts (i.e., in both cases, there was the same linear increase of RT with increasing fan). The intercept, however, was different. With general concepts, the decision took $4 \mathrm{sec}$ longer on the average than with specific concepts. The linear increase of RT seems to reflect the activation of the different number of associations that are semantic in case of the general concepts and episodic in case of the 
specific concepts. The additional and constant offset observed with general probes, on the other hand, seems to be due to the reactivation of the constant number of episodic links between the subordinated-specific concepts and the mediators.

These findings and the inherent features of the design make it most appropriate for studying predictions of the above-mentioned memory model. First, the quality as well as the quantity of the to-be-retrieved information can be varied systematically while the same retrieval cues are used: Quality is manipulated by the type of mediators, and quantity is manipulated by the level of fan. Thus, memory reactivation processes can be studied within distinct code domains, although the perceptual units that trigger these representations are the same. Second, because RT functions (and error rates) proved to be the same for different mediators, possible material-specific differences in cortical activation patterns cannot be attributed to differences in task difficulty. Finally, probes constructed from general concepts can be used as a withinsubject control condition. Although the fan effect is about the same for both specific and general concepts, only the former actually require the reactivation of an increasing number of episodically linked mediators with increasing fan. With the general concepts, on the other hand, an increase of the nominal level of fan requires the reactivation of an increasing number of a priori and semantically linked specific concepts plus the reactivation of a constant number of episodically linked mediators. Thus, a comparison of the activation patterns that accompany the processing of specific and general concepts can reveal whether both types of associative links (semantic, episodic) activate the same or different cell assemblies. Moreover, it can be tested whether or not the activation level is the same when the same number of episodic links are relevant. This should be the case with specific probes having a fan of 3 and with any of the general probes having a nominal fan of 1,2 , or 3 , because the latter always have a fan of 3 with respect to the mediators.

\section{The Measure of Cortical Activation: \\ Slow Event-Related Brain Potentials}

To monitor the activation of distinct cortical cell assemblies in human subjects, a brain imaging technique is required that possesses a high temporal and a sufficient spatial resolution; moreover, the method has to be noninvasive. Although high-spatial-resolution brain imaging techniques, such as PET or functional magnetic resonance imagery (fMRI), could be used, they have clear disadvantages for studying specific cognitive processes in normal human subjects. The signals picked up with PET or fMRI have a great inertia, they reflect metabolic and blood flow changes, and, thus, they are only indirectly (and with a certain latency) linked to the electrical activity of neuronal cell assemblies. Moreover, PET data have to be averaged over time intervals of at least $40 \mathrm{sec}$. This contrasts with the much shorter duration of functionally homogenous microstates of cognition that can be inferred from behavioral data. These seldom last lon- ger than a couple of seconds. Thus, PET measures have necessarily a restricted specificity with respect to the processes studied. They can pick up changes of brain activity related to macrostates (e.g., memory retrieval as such, or word generation vs. word understanding as such; see Raichle, 1994), but they fail when physiological correlates of microstates that last for a fraction of a second or only a few seconds (e.g., retrieval of well-defined associations in episodic memory or the processing of a semantic anomaly in a sentence) are of interest. Finally, because radioactive isotopes have to be injected for PET recordings, only very few (i.e., not more than three or four) experimental conditions can be contrasted.

One measure that better fits the criteria necessary to study microstates of cognition is provided by slow ERPs extracted from the EEG. Slow ERPs are systematic voltage shifts that prevail for at least $200 \mathrm{msec}$ but that may also last up to several seconds. According to the present state of knowledge, these slow potentials originate from activity changes in cortical cell assemblies (Creutzfeldt, 1983; Elbert \& Rockstroh, 1987; McCallum \& Curry, 1993; Speckmann, Caspers, \& Elger, 1984). Slow negative potentials seem to be due to an increase in the number and the amplitude of synchronized excitatory postsynaptic potentials (EPSPs) in the upper layers of the cortex, whereas slow positive potentials are correlated with a decrease in EPSP activity. Thus, a slow negative potential shift indicates that the activity level of a cell assembly has increased and that the assembly is in a state of higher activation or excitability. Therefore, slow ERPs provide a method to study localized changes of cortical activity. Because onset and offset of these slow ERPs can be monitored with the precision of milliseconds, the method has a much higher temporal resolution than do other currently available brain imaging methods. A disadvantage, however, is the limited spatial resolution of slow ERPs. The signals are primarily sensitive for activity changes taking place in cortical cell assemblies (Braun, Lutzenberger, Miltner, \& Elbert, 1990), and, thus, they cannot be used to monitor the full three-dimensional pattern of activity changes in both cortical and subcortical structures. For the present study, this limitation is not a real disadvantage, because the basic question concerns the functional differentiation of cortical areas during memory retrieval.

Previous work has revealed that slow ERPs have a taskspecific topography and that their amplitude is related to the effort that has to be invested into a task (e.g., W. Lang, Zilch, Koska, Lindinger, \& Deecke, 1989; Rösler, Heil, Bajrić, Pauls, \& Hennighausen, 1995; Rösler, Röder, Heil, $\&$ Hennighausen, 1993). In some studies, slow ERPs were measured while subjects were engaged in long-term memory retrieval. These revealed a parietal activation during retrieval of spatial information (Uhl et al., 1990) and a left frontal activation during retrieval of verbal information (Rösler, Heil, \& Glowalla, 1993). Thus, the findings are in line with the predictions derived from PET or lesion data (see above). However, for these two studies, it is questionable whether or not the realized tasks triggered functionally equivalent processes. Uhl et al. used an im- 
agery task with a memory retrieval component, whereas Rösler et al. tested their subjects in a variant of the fan paradigm that was less sophisticated than the one outlined above. Due to the substantial task differences of the two studies, it is difficult to tell whether the topographic differences observed in the slow ERPs were actually due to differences in the accessed memory codes. Up to now, there has been only one study in which ERPs were recorded during memory retrieval with approximately similar conditions for spatial and verbal material (Rösler, Heil, \& Hennighausen, 1995). However, in this study, too, not all of the criteria were met that permit an unequivocal conclusion with respect to the hypothesis that differences in the topography of slow ERPs are due to code-specific representations. Such an interpretation is feasible only if, except for the type of information reactivated in memory, all other aspects of the situation are held constantin particular, the difficulty of the task and the quality of the retrieval cue. This holds for the two experiments reported here.

\section{EXPERIMENT 1}

The objective of Experiment 1 was to investigate the topography of cortical activation during anticipation and retrieval of spatial information. With this material, we expected localized cortical activation over the parietal cortex during both the anticipation and the recall of the mediators. Moreover, the parietal activation should be more pronounced if more spatial information has to be retrieved (i.e., the amplitude over the parietal cortex evoked by specific concepts should increase with increasing fan). Finally, if activation of the parietal cortex is specific for the retrieval of spatial information, the nominal fan of general concepts should not influence the amplitude at this location. Since the general concepts require the retrieval of a constant number of spatial representations, the accompanying slow waves should have about the same amplitude as that evoked by specific probes having a fan of three.

\section{Method}

Subjects. Twenty-three subjects were recruited from the student population of the University of Marburg. Six subjects had to be excluded from the sample either because they did not learn the associations within $4 \mathrm{~h}$ or because their error rate in the test phase surpassed the preset limit of $15 \%$. Another 3 subjects had to be excluded because of too many artifacts in the EEG recordings. The final sample comprised 14 subjects ( 7 females, 7 males). The median age was 24 years. All subjects were right-handed and had normal or corrected-to-normal vision. All were naive with respect to the purpose of the study, and none had participated in a similar experiment before. The subjects were paid DM 10 per hour. If the error rate was less than $5 \%$ or $10 \%$, the subjects were paid an additional DM 20 or DM 10, respectively, as a bonus.

Materials. Three different types of stimuli were used: (1) Mediators were defined as locations in a grid of 18 gray squares with three rows and six columns. (2) Specific concepts were realized by means of elaborated drawings showing concrete objects. (3) General concepts were realized by means of unelaborated drawings showing the essential features of objects depicted by the specific concepts. There was a total of 18 different general concepts with one, two, or three specific concepts semantically subordinated to each (see Figure 1). General concepts were not presented before the retrieval test. Each specific concept was experimentally associated with one, two, or three mediators.

During the learning phase, only the associations between the specific concepts and the mediators were established. These associations were extensively trained. The a priori given links between general and specific concepts were taken for granted and were not explicitly learned in the course of the experiment. No direct links were established experimentally between the general concepts and the mediators.

General concepts, specific concepts, and mediators were combined in the following way: There were 18 semantic categories as represented by the unelaborated drawings serving as general concepts. This set of 18 categories was partitioned into three sets, each comprising 6 categories. In one subset, each of the 6 categories was represented by three different specific concepts, which were combined with one and only one mediator (see, e.g., the leaves in Figure 1). As a consequence, each of these specific concepts had an experimentally controlled fan of 1 (only one position associated), whereas the general concepts had an a priori given fan of 3 (three specific concepts subordinated).

For each of 6 other semantic categories, two different specific concepts were used. One of these was experimentally associated with two positions; the other one was associated with only one position (see, e.g., the butterflies in Figure 1). The six specific concepts associated with two mediators had an experimentally controlled fan of 2 , whereas the general concepts had an a priori given fan of 2 . Only the 6 specific concepts of this subgroup that were associated with two grid positions were used in the retrieval test. The associations of the other 6 specific concepts having only one link were learned but not used in the retrieval test (see Heil et al., 1994, for details of material construction).

In the third set of 6 semantic categories, each category was represented by one specific concept only. Each specific concept was associated with three positions (see, e.g., the fish in Figure 1). They define Fan 3 of the specific concepts, whereas the respective general concepts have an a priori given fan of 1 .

To summarize the fan terminology: In the case of the specific concepts, the nominal level of fan specifies the number of experimentally established associations between the specific concept(s) and the mediators. Therefore, the level of fan of the specific concepts specifies directly the amount of spatial information that has to be retrieved from long-term memory when a specific concept is used as retrieval cue. In the case of the general concepts, the nominal level of fan specifies the number of experimentally controlled a priori given (but not explicitly learned) associations that exist between the semantic categories (represented by the general concepts) and the exemplars (represented by the specific concepts). Therefore, the level of fan of the general concepts specifies the amount of pictorial information that has to be accessed on the basis of semantic associations. In addition, general concepts when used as retrieval cues trigger a constant number of spatial positions via the specific concepts. Three mediators are always associated with the total set of the specific concepts subordinated to one general concept (see Figure 1). As a consequence, a negative correlation exists between the nominal level of fan of the general concepts and the nominal level of fan of the semantically subordinated specific concepts.

As outlined in the introduction, a retrieval cue was constructed from two concepts, and the subject's task was to decide whether or not these two share a common mediator. With specific concepts, one, two, or three mediators had to be retrieved to solve the task. With general concepts, one, two, or three specific concepts had to be activated to get access to the relevant three mediators.

Both concepts forming a memory probe had the same level of fan and belonged to the same concept level (specific or general). Half of the probes were constructed from general concepts. Half of 
the trials were positive probes (i.e., the correct response was "yes"), and the other half were negative probes (with the correct answer being "no"). Test probes were selected such that the experimental factors of type of concept (specific, general), level of fan $(1,2$, or 3 ) and type of probe (positive, negative) were completely crossed. There were 30 trials for each of the resulting 12 conditions. To control for possible stimulus effects, the material was varied systematically over subjects.

Procedure. The subjects came to the lab on 2 consecutive days. On the 1st day, the subjects acquired the associations between the specific concepts and the mediators in a learning and an overlearning phase. The EEG was recorded during learning but not during overlearning. On the 2 nd day, the cued retrieval test took place with the EEG recorded.

The learning phase began after the attachment of the electrodes. The 36 specific concepts and their associated mediators were presented in random order twice while the EEG was recorded. An upright rectangle in the center of the screen was present during the whole learning phase (see Figure 2). It contained a grid of $6 \times 3$ gray squares in its upper third. Each trial was started with a bold line below the grid that was visible throughout the total trial marking the time epoch during which the EEG was recorded. It also served as a fixation aid. After a delay of $1 \mathrm{sec}$ (prestimulus baseline), an acoustic warning stimulus was presented. One second later, a specific concept appeared on the screen underneath the grid. After $4 \mathrm{sec}$, the associated position(s) turned black, while the specific concept remained visible. At the end of the EEG recording epoch (after $11 \mathrm{sec}$ ), the bold line was replaced by a thin one. The drawing-mediator combination remained visible until the subject responded with a brief upward movement of a finger that opened a light gate. The next trial was started after a variable delay of $1-2 \mathrm{sec}$. After every 6 trials, there was a short break of at least $4 \mathrm{sec}$. The subjects initiated the blocks of 6 trials by briefly lifting a finger.

In the overlearning phase, the grid of 18 squares was presented, and one specific concept appeared below. The subject had to indicate the associated spatial positions by moving the mouse-operated cursor onto the relevant position(s) and by clicking one of the mouse buttons. On this, the selected position turned black. The selection was terminated by pressing the enter key. If all correct positions had been selected, the next concept was presented. In case of an error, the correct position(s) was indicated by the computer. The subjects worked with this procedure until they made fewer than two errors in one complete set of 36 trials ( $97 \%$ correct). The sequence of drawings was varied randomly. On the average, the subjects had to work on 7-8 sets before they met the criterion. This took about $2 \mathrm{~h}$.

The test phase took place 1 day later. Three hundred sixty trials were presented as probes. Each probe was constructed from two drawings. These items were presented side by side in the center of the computer screen. The subjects responded as fast as possible, but accuracy was stressed in the instruction. The response was given by a brief upward movement of either the left or the right index finger. The allocation of the response category to the responding hand was varied systematically across subjects. The responding fingers rested in two cavities and interrupted a light gate. The subjects initiated blocks of 6 trials each by briefly lifting one of the fingers. After a delay of $2 \mathrm{sec}$ (prestimulus baseline), a warning tone was presented; $1 \mathrm{sec}$ later, the two drawings appeared on the screen. They remained visible for at least $9 \mathrm{sec}$ and were replaced by an asterisk either immediately at the end of the interval of $9 \mathrm{sec}$ (if a response had been given within this epoch) or concomitant to the response. The next trial was started after a variable delay of $750-1,750 \mathrm{msec}$. After every 6 trials, a message on the screen informed the subject about the number of errors.

The subjects were seated in a dimly lit, electrically shielded, soundattenuating room. Stimuli were presented on a cathode ray monitor (Atari SM 124; 72-Hz refresh rate). Overall luminosity of the screen was about $1.8 \mathrm{~cd} / \mathrm{m}^{2}$. The monitor was located $80 \mathrm{~cm}$ in front of the subject, with the center of the screen slightly below eye level. The drawings were $4.2 \times 4.2 \mathrm{~cm}$ (visual angle, $1.5^{\circ}$ ); the grid was 3.8 $\times 1.8 \mathrm{~cm}$ (visual angle, $1.4^{\circ} \times 0.7^{\circ}$ ). The subjects were instructed to avoid eye and body movements during the recording of the EEG. At the end of the experiment, the subjects were asked to estimate how often they had used verbal coding strategies for the mediators. All subjects reported that they had not used verbal mediators.

EEG recording, artifact handling, and signal extraction. The $\mathrm{EEG}$ was recorded monopolar with $\mathrm{AgAgCl}$ electrodes from frontopolar (Fpz), frontal (F7, F3, Fz, F4, F8), central (C3, Cz, C4), temporal (T3, T5, T4, T6), parietal (P3, Pz, P4), and occipital (O1, O2) leads, with earlobes shunted by a $5-\mathrm{k} \Omega$ resistor as reference. Eye movements and blink artifacts were monitored by means of two channels. The difference potential between two electrodes placed at the outer canthi of the two eyes provided the horizontal EOG. Two electrodes were placed below each eye and shunted with a $5-\mathrm{k} \Omega$ resistor. The difference between the signal of these two electrodes and that of an electrode placed on the central forehead below Fpz provided the vertical EOG. The left mastoid served as ground. Electrode impedance was kept below $2.5 \mathrm{k} \Omega$. Bandpass was set from direct coupling (DC) to $40 \mathrm{~Hz}$, and the digitization rate was $96 \mathrm{~Hz}$. All trials were inspected off line by one of the experimenters. Trials contaminated with artifacts were rejected. Epochs with blinks were detected by means of a Woody filter and replaced by the DC trend. Drift artifacts were corrected according to the method suggested by Hennighausen, Heil, and Rösler (1993). From the edited set of raw data, ERPs were extracted by averaging single trials separately for

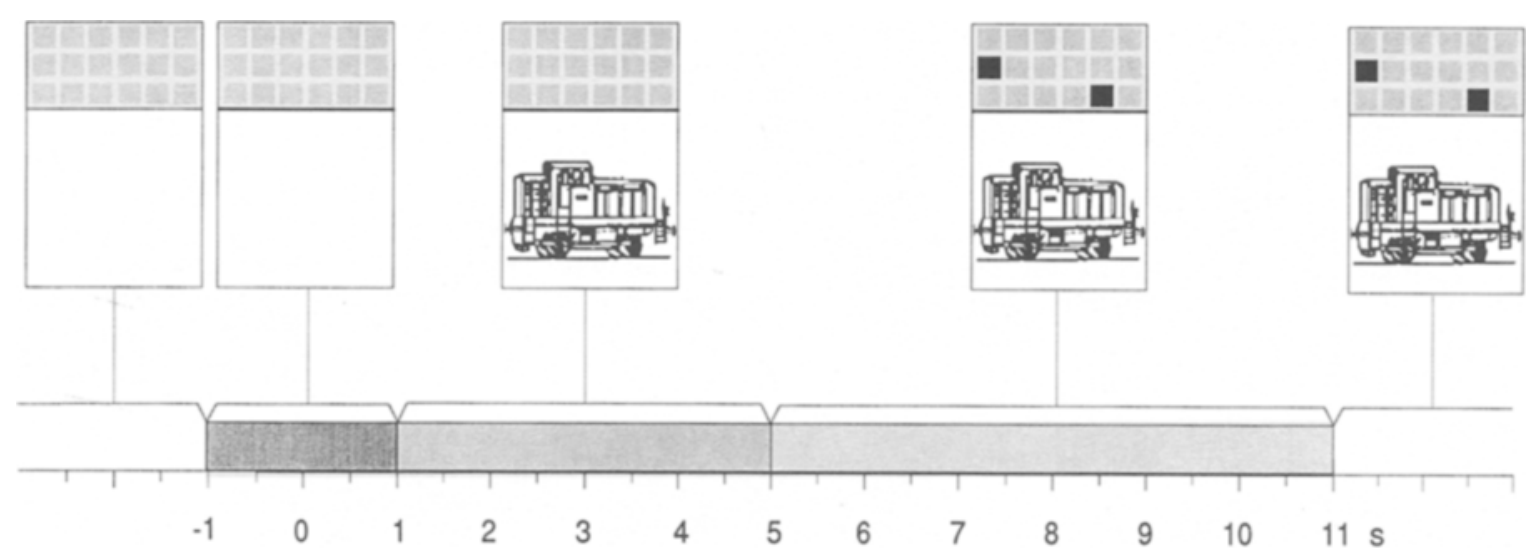

Figure 2. Time course of a trial in the learning phase. The EEG recording epoch was indicated by the thick line. The frame with the grid of gray squares (left) was present during the total learning phase. A warning stimulus was presented at 0 sec. 
subjects, electrodes, and experimental conditions. Only trials with correct responses were used for the ERP average of the test phase. The average amplitude of the epoch from 1 to $4 \mathrm{sec}$ after presentation of the drawing(s) was used as the dependent variable in both the learning and the test phase. During this interval, only drawings were presented on the screen but none of the spatial mediators. Therefore, measures of brain activity taken in this interval are not contaminated by perceptual processing of spatial information. In both cases, the subjects had to process drawings and to reactivate stored spatial representations. The amplitude was measured relative to a baseline that was defined as the average amplitude of the 1-sec interval preceding the presentation of the drawing(s).

Statistical analysis. Error rates and RTs in the test phase were each submitted to analyses of variance (ANOVAs), with factors of type of probe, type of concept, and fan.

The average amplitude of the slow ERPs of the learning phase was submitted to a two-way ANOVA, with factors of electrode and repetition (first vs. second presentation). To test the specific hypothesis of a dominant parietal activation when spatial information is reactivated, the variable "increase of the negativity from first to second presentation" was defined, and a priori contrasts were calculated such that the amplitude increase at electrode $\mathrm{Pz}$ was compared with that measured at every other electrode.

The average amplitude of the slow ERPs of the test phase was submitted to a four-way ANOVA, with factors of electrode, type of probe, type of concept, and fan. To test the specific hypothesis of a dominant parietal activation when spatial information has to be retrieved, the "variable increase of negativity from Fan 1 to Fan 3" was defined, and a priori contrasts were calculated between electrode $\mathrm{Pz}$ and every other electrode. These contrasts were run separately for specific and general concepts.

All factors were defined as within-subject effects. Significance levels of all ANOVA results were corrected according to the method of Huynh and Feldt (1976) to compensate for nonsphericity of the data.

\section{Results}

RTs and error rates. The error rates were reliably influenced only by type of concept $[F(1,13)=18.15, p<$ $.01]$. The subjects committed more errors $(10.5 \%)$ when they were probed with two general concepts whose associations had not been learned explicitly than when they were probed with two specific concepts $(5.1 \%)$.

Mean RTs and their standard deviations are given in Table 1. They are also summarized graphically in Figure 3. These results replicate the results of Heil et al. (1994). RT was found to be a function of the number of associative links fanning out from a given memory representation [fan, $F(2,26)=35.61, p<.01]$. This functional relationship held for both types of links that had to be activated: the a priori given associations between general and specific concepts and the experimentally established associations between specific concepts and grid locations [interaction type of concept $\times$ fan, $F(2,26)=0.20$ ] Nevertheless, decisions on the relatedness of two general concepts took $2.5 \mathrm{sec}$ longer, on average, than did decisions on the relatedness of two specific concepts [type of concept, $F(1,13)=61.19, p<.01]$. For positive probes, the fan effect for general concepts was less pronounced, resulting in a reliable main effect of type of probe $[F(1,13)$ $=35.13]$ and reliable interactions with type of probe [type of probe $\times$ type of concept, $F(1,13)=28.15$; type of probe $\times$ fan, $F(2,26)=7.37$; type of probe $\times$ type of concept $\times$ fan, $F(2,26)=11.67$; all $p s<.01)$ ].

Slow ERPs in the learning phase. The time course and the topography of the slow ERPs evoked by the first and the second presentation of the drawings are presented in Figure 4. As can be seen, the onset of the drawing at $1 \mathrm{sec}$ initiated a very pronounced negativity over the posterior part of the scalp (electrode row T5 to T6, and electrodes $\mathrm{O} 1$ and $\mathrm{O} 2$ ). The average amplitude measured in the interval after the presentation of the drawing and before the presentation of the mediators $(2-5 \mathrm{sec})$ was submitted to an ANOVA, with factors of electrode and repetition (first vs. second presentation). This analysis revealed a reliable main effect of electrode $[F(17,221)$ $=35.30, p<.01]$ and a marginal main effect of repetition $[F(1,13)=3.97, p=.0677]$. Most interesting, however, was the reliable interaction $[F(17,221)=10.55$, $p<.01]$. Electrode-specific comparisons revealed a reliable increase in the negativity with repetition at electrode positions $\mathrm{C} 3, \mathrm{~T} 5, \mathrm{P} 3, \mathrm{Pz}, \mathrm{P} 4, \mathrm{O} 1$, and $\mathrm{O} 2[F \mathrm{~s}(1,13)=$ $15.83,11.18,35.50,22.92,17.99,17.58$, and 12.27 , respectively, all $p \mathrm{~s}<.01]$. Pairwise comparisons of this increase in negativity between $\mathrm{Pz}$ and the other electrodes revealed that the increase at $\mathrm{Pz}$ was higher than the increase at any other electrode except $\mathrm{P} 3$, the direct neighboring position. All of these effects of a topographic difference proved to be reliable when the mean amplitudes were standardized such that level and dispersion differences between conditions and subjects were eliminated (McCarthy \& Wood, 1985). ${ }^{1}$

Slow ERPs in the test phase. The slow ERPs measured during retrieval of information in a cued recall test are presented in Figures 5 and 6 with specific and gen-

Table 1

Effects of Experimental Conditions on

Mean Response Times (in Milliseconds) and Standard Deviations

\begin{tabular}{|c|c|c|c|c|c|c|c|c|c|c|c|c|}
\hline \multirow[b]{3}{*}{ Probe } & \multicolumn{6}{|c|}{ General Concepts } & \multicolumn{6}{|c|}{ Specific Concepts } \\
\hline & \multicolumn{2}{|c|}{ Fan 1} & \multicolumn{2}{|c|}{ Fan 2} & \multicolumn{2}{|c|}{ Fan 3} & \multicolumn{2}{|c|}{ Fan 1} & \multicolumn{2}{|c|}{ Fan 2} & \multicolumn{2}{|c|}{ Fan 3} \\
\hline & $M$ & $S D$ & $M$ & $S D$ & $M$ & $S D$ & $M$ & $S D$ & $M$ & $S D$ & $M$ & $S D$ \\
\hline \multicolumn{13}{|c|}{ Experiment 1 (Spatial Mediators) } \\
\hline Negative & 7,718 & 2,502 & 9,561 & 3,047 & 10,826 & 3,192 & 4,668 & 2,012 & 5,470 & 1,823 & 6,553 & 2,109 \\
\hline Positive & 6,943 & 2,224 & 7,588 & 2,778 & 7,543 & 2,402 & 4,323 & 1,807 & 5,614 & 2,036 & 6,295 & 1,830 \\
\hline \multicolumn{13}{|c|}{ Experiment 2 (Verbal Mediators) } \\
\hline Negative & 9,211 & 1,830 & 9,884 & 2,451 & 11,330 & 2,669 & 4,237 & 1,511 & 5,988 & 1,264 & 7,995 & 1,672 \\
\hline Positive & 6,836 & 2,205 & 7,369 & 2,524 & 7,821 & 3,318 & 3,607 & 1,072 & 5,152 & 1,598 & 5,993 & 1,888 \\
\hline
\end{tabular}




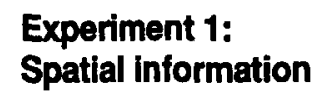

\section{Experiment 2: Verbal information}
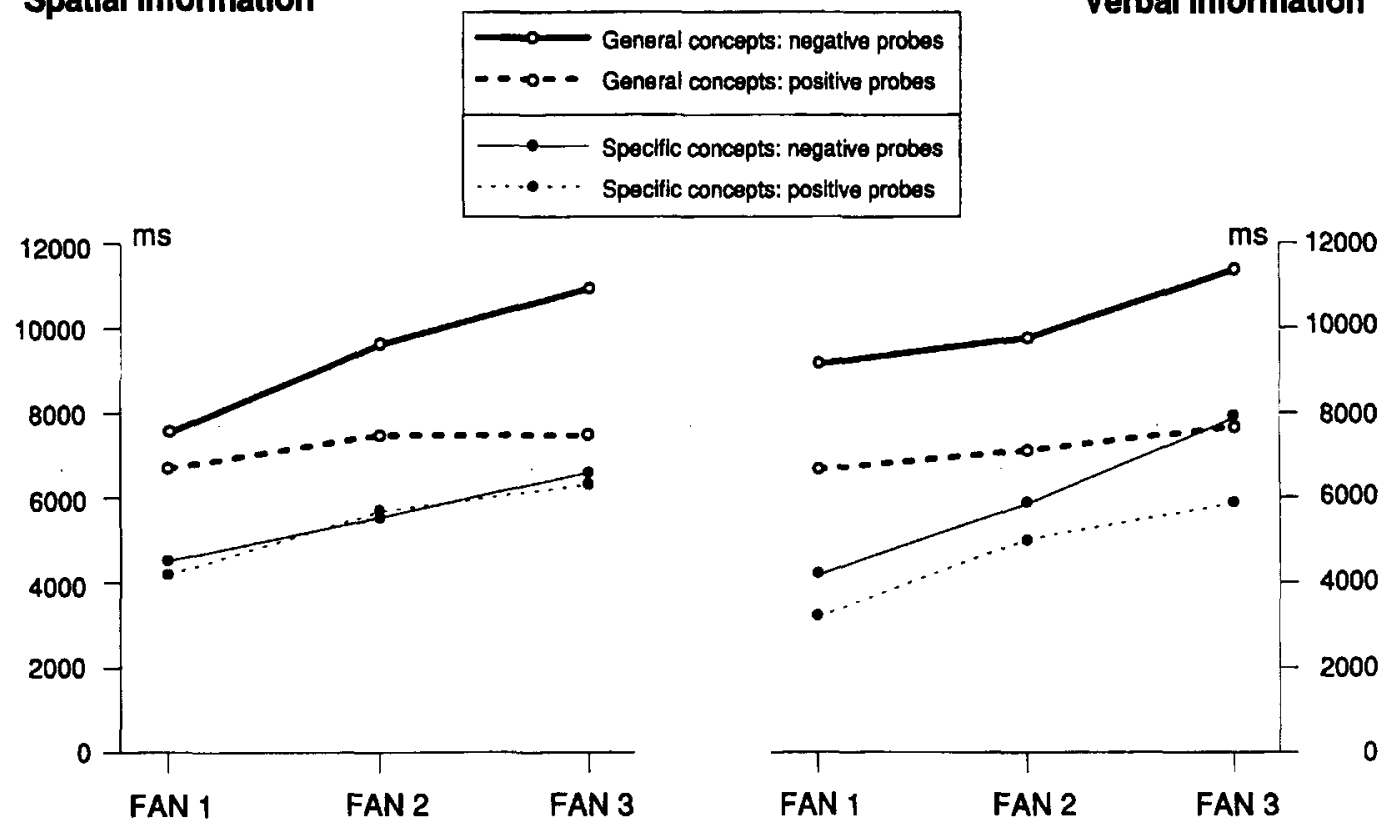

Figure 3. Mean response times as a function of type of concept and level of fan for positive and negative probes in Experiments 1 and 2.

eral concepts, respectively, as retrieval cues. A slow negative shift over the posterior part of the scalp was discernible in all six conditions. It developed shortly after stimulus onset and prevailed for several seconds. The maximum of this negative-going potential was found over the parietal cortex.

An ANOVA revealed a very pronounced main effect of electrode $[F(17,221)=92.03 ; p<.01]$ and a reliable interaction of electrode $\times$ type of probe $[F(17,221)=$ $5.86, p<.01]$. The latter reflects that topographic differences were somewhat more pronounced for negative probes than for positive probes. The reliable three-way interaction of electrode $\times$ type of concept $\times$ fan $[F(34,442)$ $=3.15, p<.01]$ reflects the amplitude modulation over the parietal cortex caused by the varying level of fan of specific concepts. With specific concepts, the slow negative potential over the parietal cortex was the higher the more spatial information had to be retrieved from episodic long-term memory (see Figure 5). Electrode-specific analyses revealed a reliable increase of the negative potential with increasing fan at electrodes $\mathrm{C} 3, \mathrm{C4}, \mathrm{P3}, \mathrm{Pz}$, and P4 $\left[F_{\mathrm{s}}(2,26)=10.16,5.48,13.92,20.13\right.$, and 4.60 , respectively, all $p s<.05]$. Pairwise comparisons revealed that at $\mathrm{Pz}$ the amplitude increase from Fan 1 to Fan 3 was higher than at any other electrode.

With general concepts (Figure 6), on the other hand, the amplitude of the slow wave was not affected at any electrode by the nominal level of fan (all contrasts provide $F$ ratios with $p>.25$ ). Obviously, activating one, two, or three specific concepts on the basis of a priori given semantic knowledge does not become manifest in a slow negative potential shift. Nevertheless, a pronounced parietal activation was present for these types of probes. It had the same amplitude and topography for all three levels of semantic fan, and the amplitude was of about the same size as observed with specific probes having an episodic fan of 3 .

\section{Discussion}

The pattern of RTs observed in this experiment substantiates the claim of Heil et al. (1994) that the same temporal dynamics hold for memory retrieval processes, irrespective of whether the accessed links are episodic and experimentally trained or semantic and a priori given. In both cases, activation seems to spread across links with the same speed and dissipation characteristic.

A summary of the ERP results of Experiment 1 is given graphically in Figure 7. The voltage-time plots clearly reveal a restricted activation of the parietal cortex during access of spatial memory contents. The effect is disclosed (1) in the learning phase while the subjects anticipate spatial information and (2) in the test phase while spatial associations have to be reactivated in a cued recall test. In both situations (anticipation and recall) and with both types of retrieval cues (specific and general concepts), the maximum negative amplitude is found at $\mathrm{Pz}$. Moreover, with specific probes the amplitude at $\mathrm{Pz}$ increases systematically with increasing fan. This amplitude increase implies a more pronounced cortical activation when more spatial associations have to be reacti- 


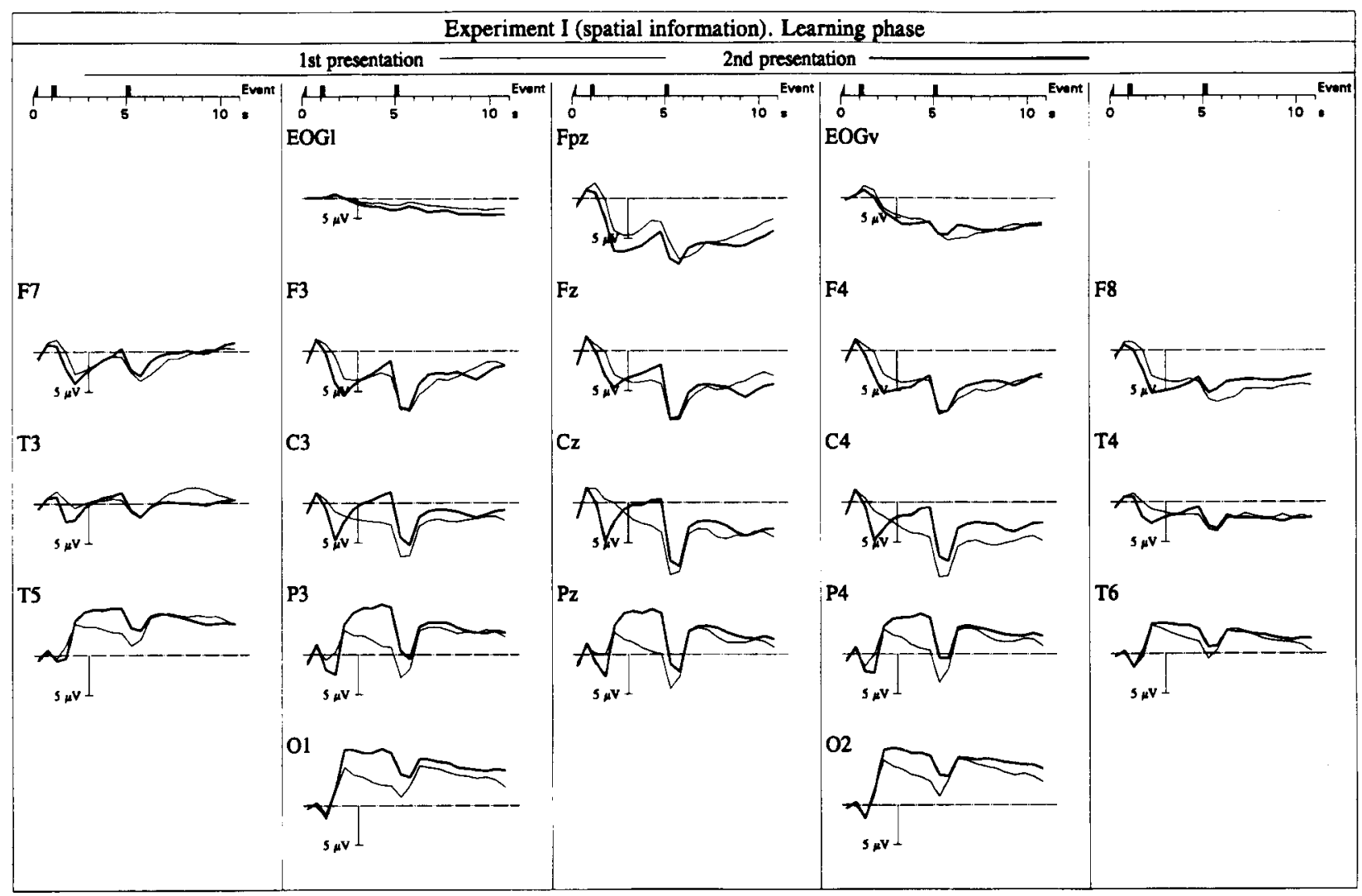

Figure 4. Grand average of the slow-wave activity obtained during the learning phase of Experiment 1. Thin line $=$ first presentation of the specific concepts. Thick line $=$ second presentation of the specific concepts. The time scale started with the presentation of the warning stimulus. The specific concept (a drawing) was presented at 1 sec, the mediator(s) was added at 5 sec. Negativity was up.

vated. With general probes, the amplitude at Pz is constant for all three levels of fan, and it has about the same size as with specific probes having a fan of 3 . This suggests that the activation that becomes manifest in the slow-wave amplitude over the parietal cortex is specific for the activation of episodic associations in memory.

The slow-wave effect observed at Pz cannot be attributed to a general increase of effort (Kahnenman, 1973) or an allocation of unspecific resources (Wickens, 1984). This becomes evident if the effects observed in the two domains of dependent variables (RTs and the slow waves) are compared with each other. RTs increased with increasing fan for both types of concepts, and they were substantially longer for general concepts than for specific concepts. Thus, on the basis of the RTs, one would have to conclude that the task becomes more difficult from Fan 1 to Fan 3 with both types of probes and that it is more difficult with general concepts. The amplitude of the slow wave recorded over the parietal cortex, on the other hand, reveals a different pattern of results. It is monotonically related only to the number of episodic associations that have to be activated in spatial memory (one, two, or three mediators with specific concepts, and always three mediators with general concepts). In contrast, the number of semantic links that bears a clear effect on RTs is not re- flected in any of the amplitude measures. Therefore, one can conclude that the amplitude of the slow brain potential over the parietal cortex is specifically related to the retrieval of spatial information from episodic long-term memory, not to an unspecific amount of effort invested in the task. Moreover, the activation that becomes manifest in the parietal slow wave is not related to the reactivation of a priori given semantic knowledge.

The present set of data does not provide any evidence that memory retrieval of spatial information goes together with a lateralization of activation over the right hemisphere. This is consistent with findings reviewed by Ehrlichman and Barrett (1983), which converge to the conclusion that mental imagery is a bilaterally shared function. Kosslyn, Holtzman, Gazzaniga, and Farah (1985) found that the left hemisphere might be more important than the right if spatial representations have to be processed with high resolution. Thus, our finding of an equal distribution of activation over both hemispheres in a spatial task is not at variance with the literature (see also Kosslyn, 1987). Finally, the claim that both parietal hemispheres might be equally activated in our experiment because of a "verbal mediation" of the task will lose its impact in light of the results of Experiment 2. The activation of verbal information in episodic long-term mem- 


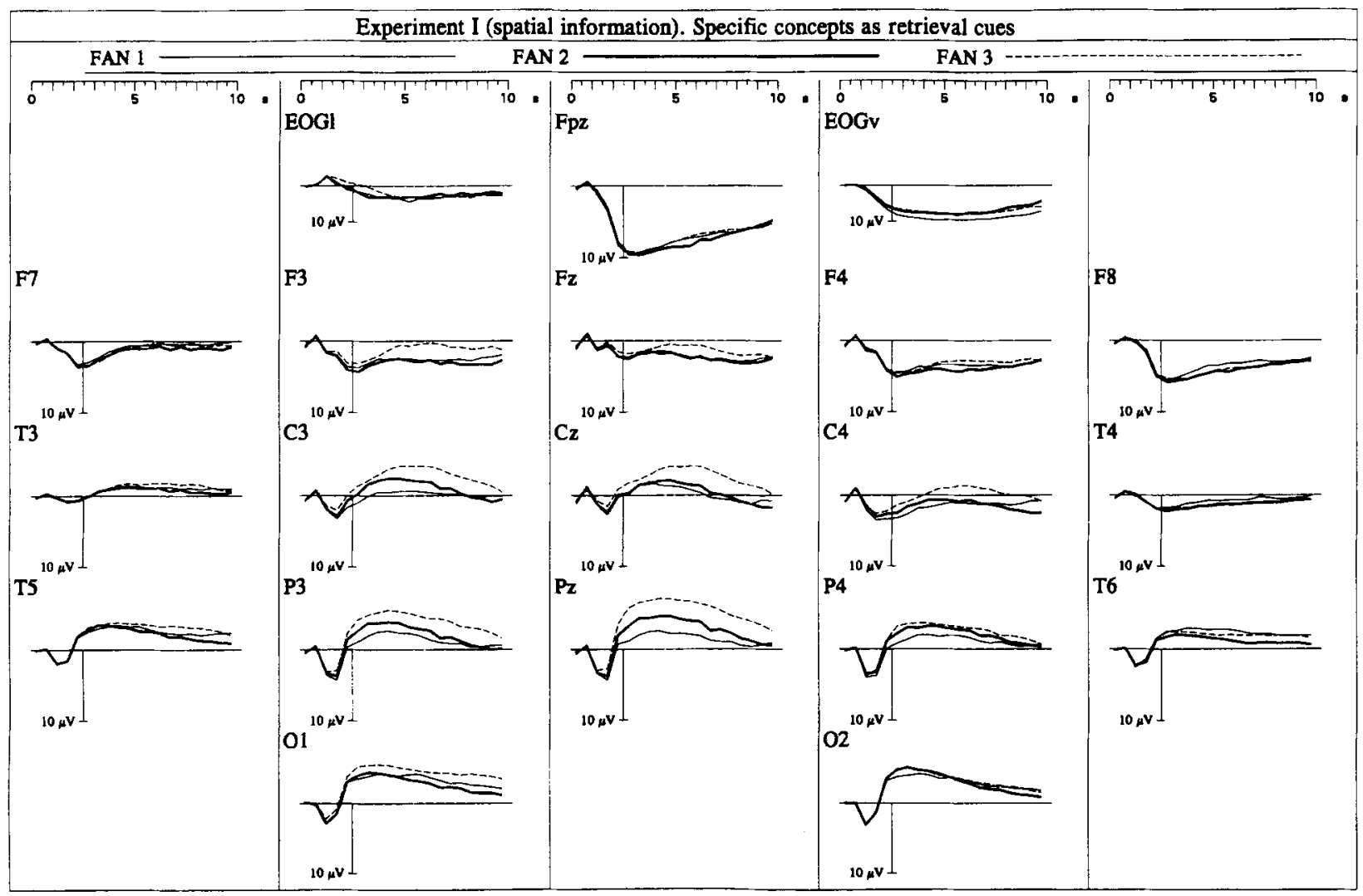

Figure 5. Test phase of Experiment 1. Grand average of the slow-wave activity obtained with specific concepts for different levels of fan. The probe pair was presented at 1 sec.

ory results in a topographically restricted activation of the left frontal cortex, and not just a general lateralization to the left hemisphere.

\section{EXPERIMENT 2}

The results of Experiment 1 provided strong evidence for an activation of the parietal cortex during the retrieval of spatial information from episodic long-term memory. The retrieval of verbal information, on the other hand, should be accompanied by an activation of the left frontal cortex. In principle, such an effect was already revealed in a previous study by our group (Rösler, Heil, \& Hennighausen, 1995); however, the results of that study cannot be compared directly with those of Experiment 1. In the previous study, both targets and mediators were realized by nouns. Therefore, the left frontal activation that was observed in the present Experiment 1 could have been due to the processing of the verbal retrieval cue, the access of the verbal mediators, or both. To find clear evidence for our hypothesis that the negative potential over the left frontal cortex is specifically related to verbal memory retrieval, as the parietal negativity was found to be specifically related to spatial memory retrieval, Experiment 2 was constructed as a complete mirror image of Experiment 1. In particular, the number of items, the number of associations, the learning and the overlearning procedure, the criterion, the retrieval cues, and the retrieval test itself were completely equivalent. Only one feature was changed: Instead of grid positions, concrete nouns served as mediators.

\section{Method}

Subjects. Twenty-one subjects were recruited from the student population of the University of Marburg. Four subjects had to be excluded from the sample either because they did not learn the associations within $4 \mathrm{~h}$ or because their error rate in the test phase had surpassed the preset limit of $15 \%$. Another 3 subjects had to be excluded because of too many artifacts in the EEG recordings. The final sample comprised 14 subjects ( 9 females, 5 males). The subjects' median age was 25 years. All subjects were right-handed and had normal or corrected-to-normal vision. All were naive with respect to the purpose of the study, and none had participated in a similar experiment before. Modus of payment was the same as in Experiment 1.

Materials. Exactly the same elaborated and unelaborated drawings as were used in Experiment 1 were used as specific and general concepts. Eighteen concrete nouns were used as mediators (e.g., lawyer, airport, and radio). These nouns were not semantically associated with the drawings (for details, see Heil et al., 1994). All other aspects of the material were the same as before. In particular, the experimental factors of level of fan and type of concept were realized in the same manner. Specific drawings were associated with one, two, or three concrete nouns, and their general counterparts had one, two, or three specific concepts subordinated. The sum of the 


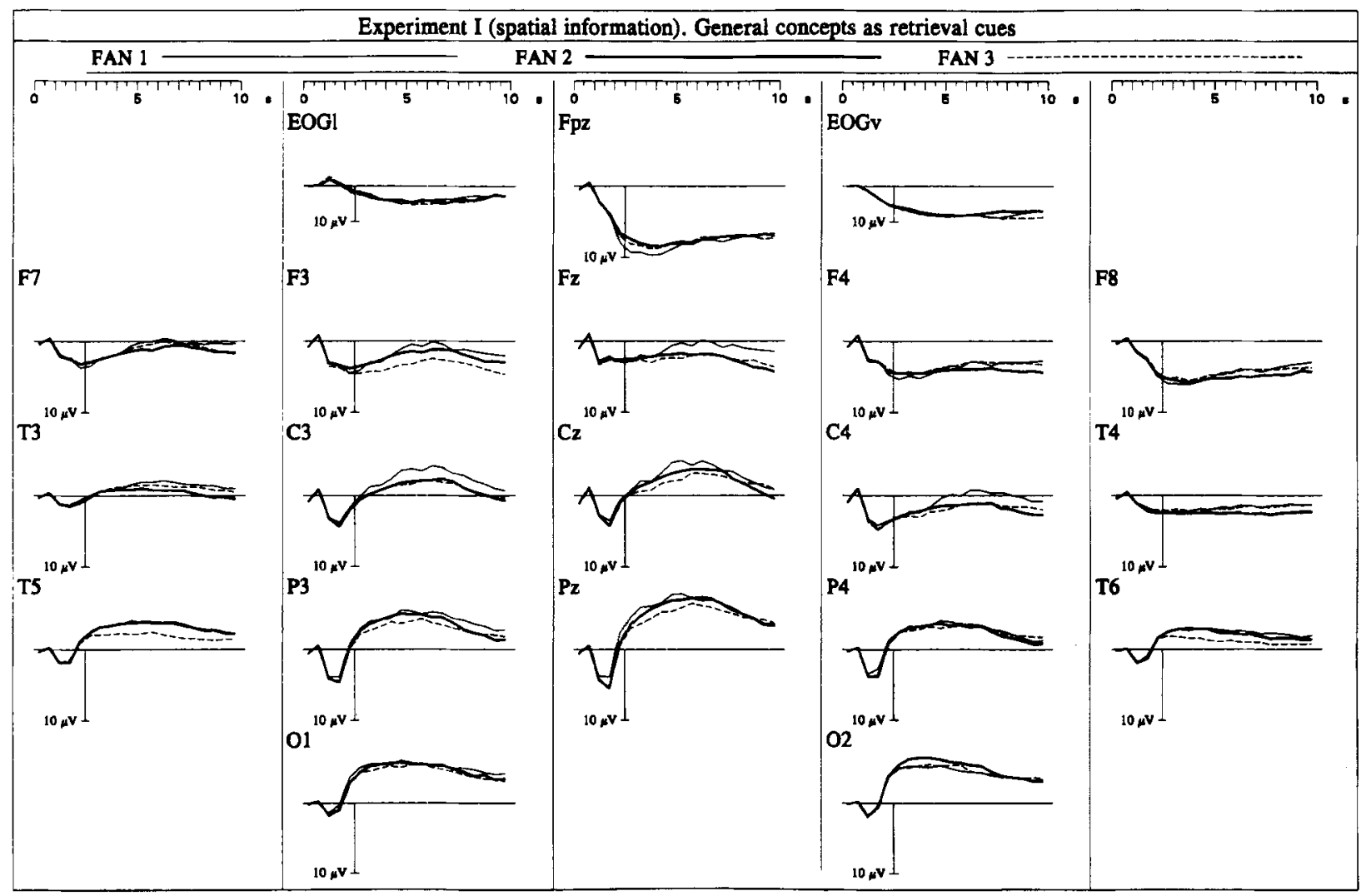

Figure 6. Test phase of Experiment 1. Grand average of the slow-wave activity obtained with general concepts for different levels of fan.

mediators associated to all specific concepts being subordinated to a given general concept was again constant (i.e., three).

Procedure. Learning, overlearning, and testing were identical to the procedures described in Experiment 1. During acquisition, the concrete nouns appeared above the drawing in the same section of the display where the positions had been shown in Experiment 1. The sequence of the nouns within the frame was varied randomly to avoid any spatial cues. EEG recording, signal extraction, and the statistical analyses were also the same as before.

\section{Results}

RTs and error rates. When probed with general concepts, the subjects committed more errors with positive probes $(10.6 \%)$ than with negative probes $(3.1 \%)$. An equivalent effect was not found for the specific concepts, whose associations to the mediators had been experimentally trained (the respective error rates for positive and negative probes were $4.5 \%$ and $2.4 \%$ ). Accordingly, an ANOVA revealed reliable main effects of type of concept $[F(1,13)=24.95]$ and type of probe $[F(1,13)=29.10]$ and a reliable interaction of these two factors $[F(1,13)=$ 9.73] (all $p$ s $<.01$ ).

Mean RTs and their standard deviations are given in Table 1 and are also summarized graphically in Figure 3. These results clearly replicate the results of Heil et al. (1994), although, here, drawings, and not words, served as retrieval cues.

Again, RTs were longer for negative probes than for positive probes $[F(1,13)=43.06, p<.01]$ and were longer for general concepts than for specific concepts $[F(1,13)=$ $98.51, p<.01]$. As before, RT proved to be a function of the number of associative links fanning out from a given memory representation [main effect of fan, $F(2,26)=$ $75.77, p<.01]$. This increase in RT with increasing fan was more pronounced for specific concepts than for general concepts [interaction type of concept $\times$ fan $[F(2,26)$ $=5.86, p<.05]$. A comparison of positive and negative probes revealed that the fan effect was less pronounced for positive probes than for negative probes [interaction type of probe $\times$ fan, $F(2,26)=8.15, p<.01]$. Likewise, the difference in the speed of access to the mediators via general and specific concepts was less distinct for positive probes than for negative probes [interaction type of probe $\times$ type of concept, $F(1,13)=13.82, p<.01]$. Overall, however, the pattern of results was, by and large, the same for positive and negative probes (see Figure 3).

Slow ERPs in the learning phase. The slow ERPs evoked by the first and the second presentation of the drawings are shown in Figure 8. As can be seen, the repetition of the target stimuli in the learning phase resulted in an increase of a slow negative potential over the left and the central part of the scalp. This effect was most prominent in the interval between onset of the drawing and onset of the mediators (i.e., it emerged while the subject anticipated the associated mediators) [main effect of repetition, $F(1,13)=5.21, p<.05$; interaction of electrode position $\times$ repetition, $F(17,221)=6.84, p<.01]$. 


\section{Anticipation}
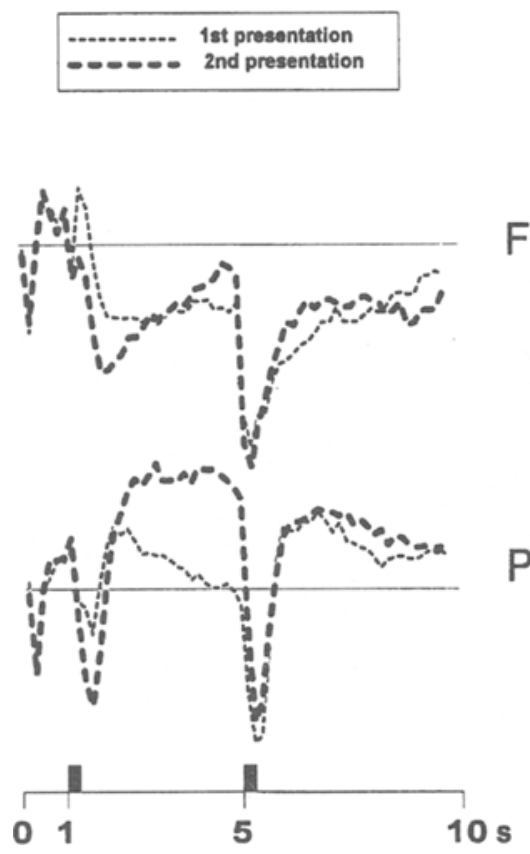

\section{Spatial information}

\section{Retrieval}

specific probes

general probes

F3
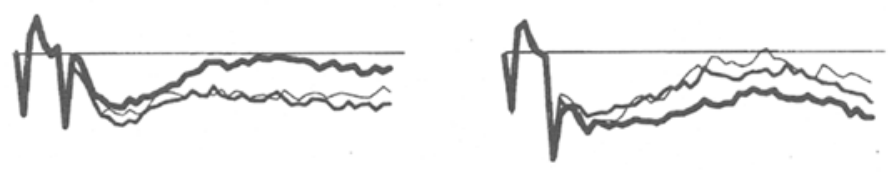

Pz

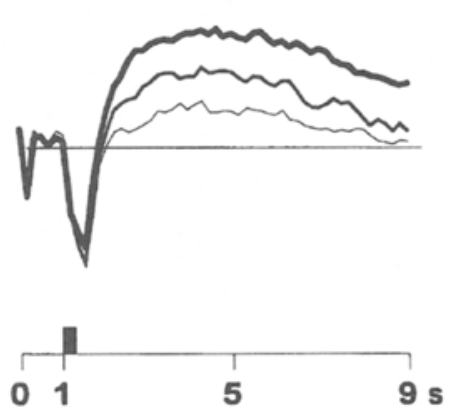

Figure 7. Summary of the main results of Experiment 1 for electrode positions F3 (left frontal) and Pz (mid-parietal).

Electrode-specific comparisons between the first and the second presentation revealed a reliable increase in the negativity over the left frontal and the central cortex [positions $\mathrm{F} 7, \mathrm{~F} 3, \mathrm{Fz}, \mathrm{C} 3$, and $\mathrm{Cz}, F(1,13)=7.04,5.58,4.00$, 15.69 , and 15.19 , respectively, all $p s<.05$ ], which was predicted by the hypotheses. In addition, the negativity increased reliably over the central parietal and the right occipital cortex $[\mathrm{Pz}$ and $\mathrm{O} 2, F(1,13)=20.65$ and 11.37, respectively, both $p s<.01]$.

Slow ERPs in the test phase. The time course and the topography of the slow ERPs evoked during the retrieval of verbal information are presented in Figures 9 and 10 with specific and general concepts as retrieval cues, respectively. As can be seen, the presentation of the retrieval cue initiated a pronounced negativity over the left frontal cortex in all conditions [main effect electrode, $F(17,221)=61.12]$.

More informative, however, is the reliable three-way interaction of electrode $X$ type of concept $\times$ fan $[F(34,442)$ $=2.34, p<.02]$. It reflects the fact that the fan manipulation was effective only with specific concepts and at electrodes placed over the left frontal cortex [two-way interaction of electrode $\times$ fan calculated separately for specific concepts, $F(34,442)=2.66, p<.01]$. The negative amplitude over the left frontal cortex was higher, the more verbal information had to be retrieved from episodic long- term memory (see Figure 9). Electrode-specific analyses of the fan manipulation revealed a reliable increase of the negativity at electrode positions Fpz, F3, Fz, and $\mathrm{F} 4[F s(2,26)=11.69,6.20,6.61$, and 5.60, respectively, all $p s<.05]$. Pairwise comparisons revealed that the increase in negativity at $\mathrm{F} 3$ was higher than the increase at any other electrode.

As in Experiment 1, the amplitude of the slow waves was not affected by the fan manipulation when general concepts were used as retrieval cues (see Figure 10). The reactivation of one, two, or three drawings on the basis of semantic knowledge did not become manifest in the psychophysiological data [interaction of electrode $\times$ fan calculated separately for general concepts, $F(34,442)=$ 0.99 ]. A left frontal activation, however, was present for these probes.

\section{Discussion}

The RTs observed in Experiment 2 revealed a clear and substantial fan effect for negative and positive probes, as well as for specific and general concepts. Thus, RT proved again to be a function of the amount of information that had to be reactivated in long-term memory. This relationship held, irrespective of whether semantic or episodic links had to be accessed and irrespective of whether the final decision was "yes" or "no." This repli- 


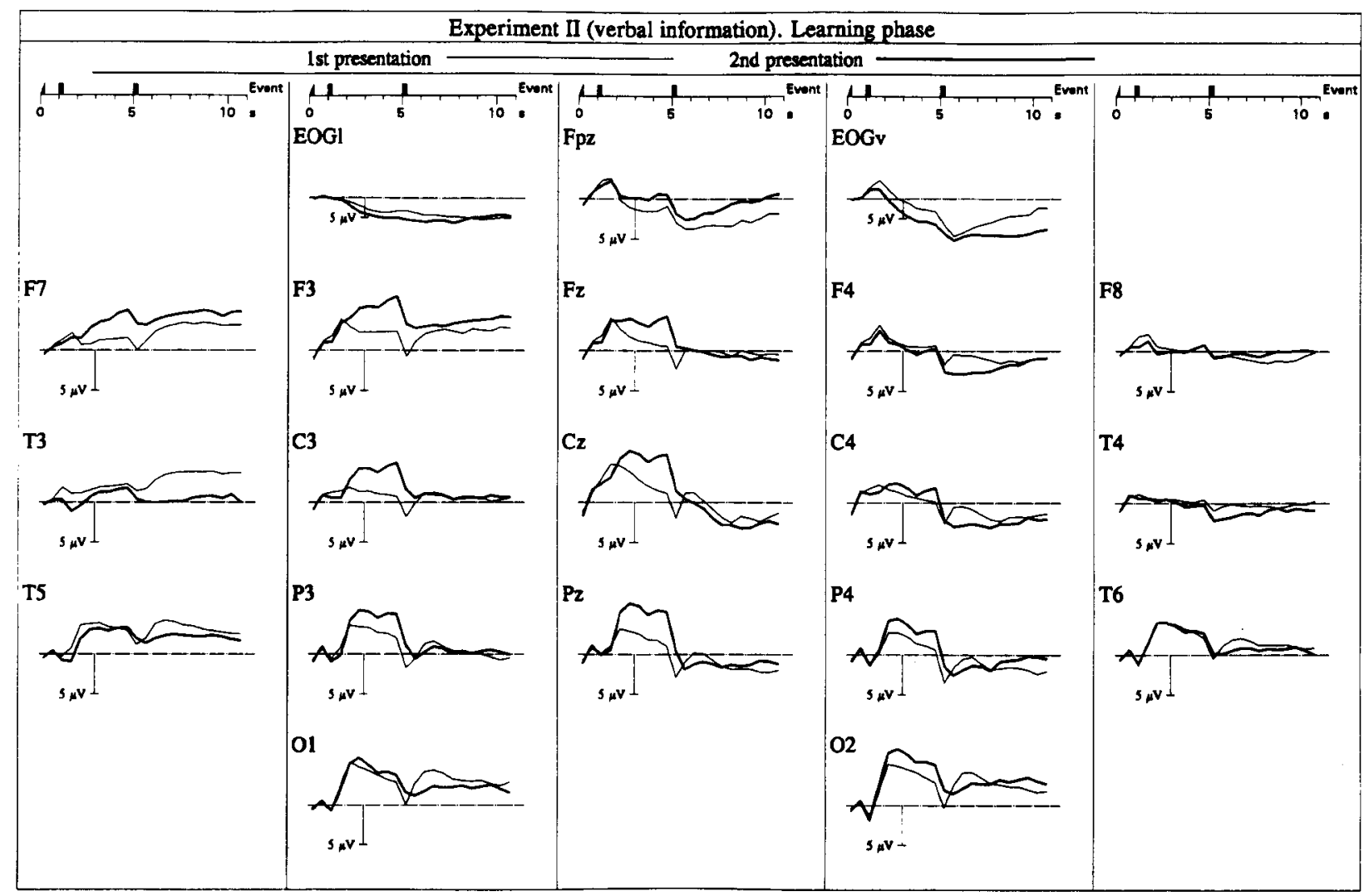

Figure 8. Grand average of the slow-wave activity obtained during the learning phase of Experiment 2. Verbal mediators were presented at 1 sec.

cates the findings of Heil et al. (1994), although now drawings served as retrieval cues and the retention interval extended over 1 day instead of only $1 \mathrm{~h}$.

The overall pattern of RTs observed with verbal mediators was also, by and large, the same as in Experiment 1 with spatial mediators. Nevertheless, one has to admit that when general concepts served as retrieval cues, the fan effect followed a somewhat different trend with spatial and verbal mediators. This somewhat weakens the general thesis that the temporal dynamics of memory activation processes are exactly the same for all kinds of codes. On the other hand, there is sufficient evidence from this experiment and from previous studies to conclude that decision time during memory retrieval depends on the amount of information that has to be reactivated or scanned. A linear increase of RT with an increasing number of associated representations was found with any kind of probe and with all three types of links involved: episodic spatial (Experiment 1), episodic verbal (Experiment 2), and semantic links (general concepts, both experiments). The same was also found in the experiments of Heil et al. (1994) and Rösler et al. (1995) in which comparable experimental setups had been realized. Thus, the findings of all of these studies clearly suggest that very similar, if not the same, functional principles must be valid if differently coded representations are reactivated.
The ERP results of Experiment 2 are summarized graphically in Figure 11. They clearly reveal an activation of the left frontal cortex during access of verbal memory contents. This effect became manifest again in the learning phase while the subjects anticipated verbal mediators and in the retrieval phase while these mediators were reactivated by a cue. In its essence, the pattern of results is the same as that found with spatial mediators in Experiment 1 (cf. Figure 7). The only, but substantial difference concerns the topography of the effects in the retrieval test. In Experiment 1 with spatial mediators, the maximum of the negative slow wave was found over the parietal cortex. In Experiment 2 with verbal mediators, the maximum of the negative slow wave was recorded over the left frontal cortex (F3), whereas the amplitude over the parietal cortex $(\mathrm{Pz})$ was much smaller. At F3, where the maximum negativity was recorded, the amplitude was also monotonically related to the number of the to-bereactivated mediators-i.e., one, two, or three concrete nouns for the specific concepts and always three concrete nouns for the general concepts). The amplitude level at F3 reflects exactly this ordinal relationship -it was smallest for specific Fan 1 probes, intermediate for specific Fan 2 probes, and largest, and of about the same size, for both specific Fan 3 probes and all three general probes. 


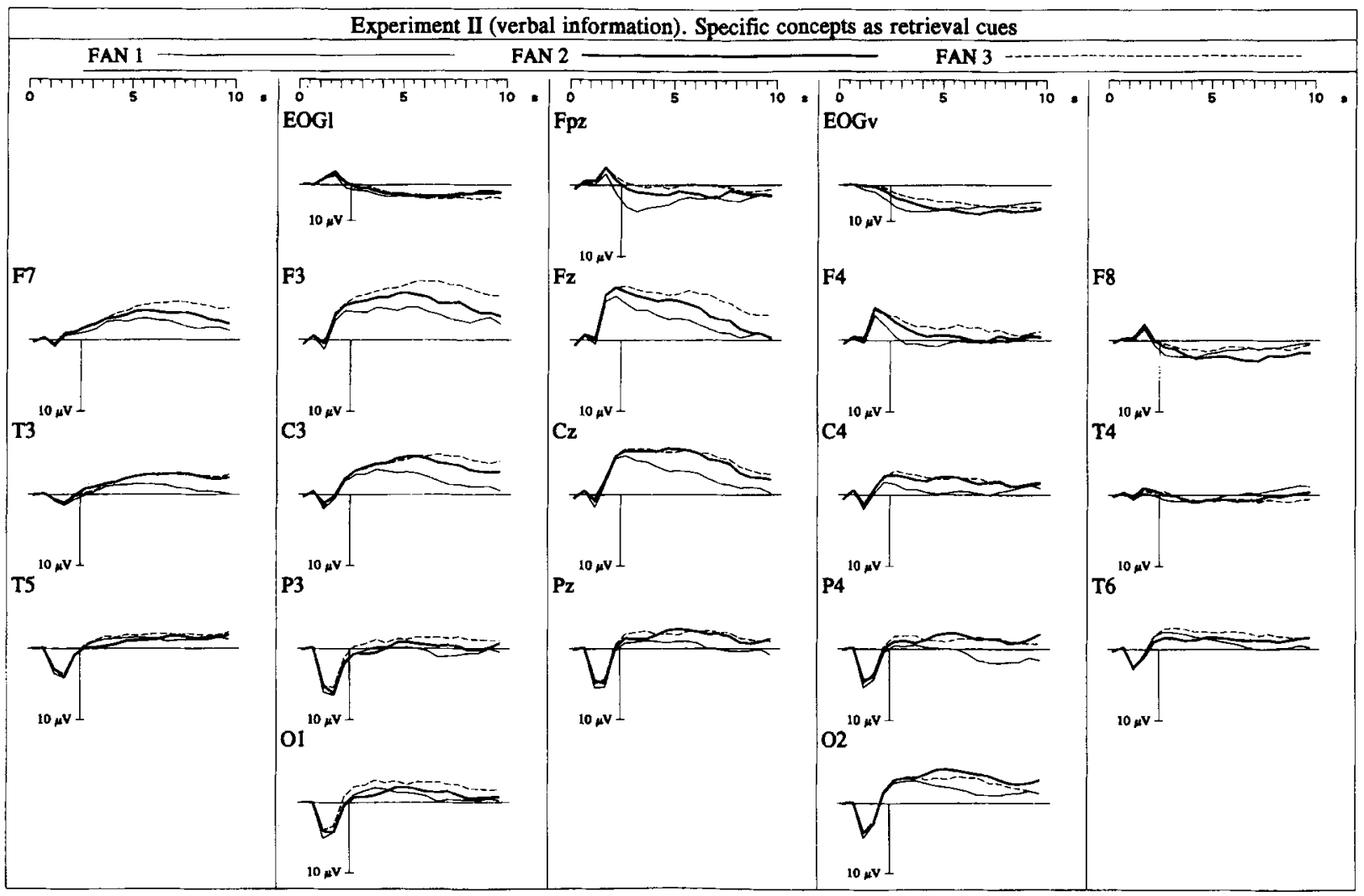

Figure 9. Test phase of Experiment 2. Grand average of the slow-wave activity obtained with specific concepts for different levels of fan.

The pattern of findings again excludes a less specific explanation that attributes the slow-wave effects simply to differences in the overall difficulty of the task or, in other words, to differences in the amount of effort that has to be invested by the subject. As with spatial mediators, the RTs observed with verbal mediators were affected by two factors: the number of experimentally trained mediators that had to be reactivated (Fan 1, 2, and 3 of the specific concepts) and the number of specific concepts that were linked to the general concepts on the basis of a priori knowledge (Fan 1, 2, and 3 of the general concepts). The amplitude level at F3, on the other hand, was affected only by the number of experimentally trained verbal links. Thus, RT obviously reflects the overall difficulty of the task, whereas the amplitude of the slow wave over the left frontal cortex reflects only one particular aspect of memory retrieval (i.e., the reactivation of episodic associations established between the target items and the verbal mediators).

There is only one finding in this experiment that deviates from our predictions. In the learning phase, when verbal mediators could be anticipated (second presentation of the stimulus material), an increase of the slowwave amplitude was registered not only over left frontal but also over central and parietal areas (cf. Figure 7). Since such an activation of central and parietal areas was not present during the retrieval test proper, it seems unlikely that this effect is a specific correlate of processes that reactivate verbal material. In several studies, it was found that a negative slow wave with a central to parietal topography appeared in situations in which the subject could anticipate task-relevant information but in which this information was very likely not coded as a verbal representation (Brunia, 1988; Brunia \& Damen, 1988; Rösler \& Heil, 1991). Thus, it could be that, in the learning phase of the present study, two negative slow waves with different generators prevailed in the EEG: one specifically related to the reactivation of verbal mediators (maximum at F3) and one related to the anticipation of task-relevant information as such (maximum at Pz). In Experiment 1 with spatial mediators, both processes could not show up as distinct waves, because the topography of the slow wave specifically related to the reactivation of spatial mediators also had a parietal maximum. Further studies will be necessary to disentangle the various processes that might prevail during stimulus anticipation.

Irrespective of this caveat, it can be concluded that the left frontal negative slow wave is specifically related to a functional microstate of the brain that prevails when verbal episodic memory contents have to be accessed. This conclusion is supported by two facts. First, the left frontal negativity appears whenever verbal memory con- 


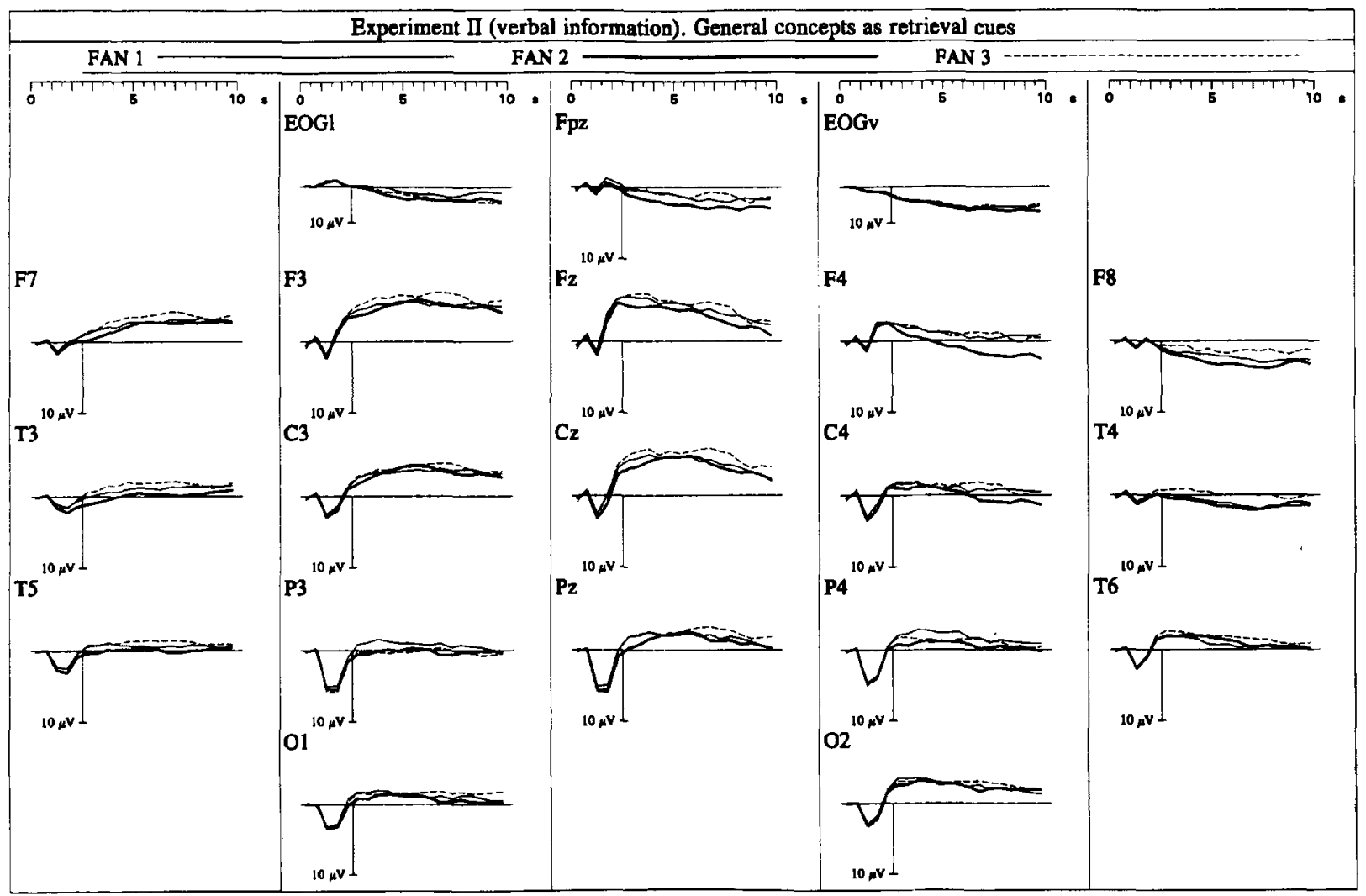

Figure 10. Test phase of Experiment 2. Grand average of the slow-wave activity obtained with general concepts for different levels of fan.

tents have to be reactivated (it is present during both anticipation learning and retrieval of verbal mediators). Second, the amplitude of the left frontal negativity is monotonically related to the number of verbal mediators that have to be reactivated in the retrieval test.

\section{DIRECT STATISTICAL COMPARISON OF EXPERIMENTS 1 AND 2}

On the basis of within-experiment ANOVAs, all effects proved reliable when both experiments were combined into one split-plot ANOVA with an additional betweensubject factor of experiment. Most important, these overall analyses revealed, for the learning phase, a reliable three-way interaction of experiment $x$ electrode $\times$ repetition $[F(17,442)=5.37, p<.01]$ and, for the retrieval test, a reliable four-way interaction of experiment $\times$ electrode $\times$ type of concept $\times$ fan $[F(34,884)=3.21, p<$ $.01]$. The distinct overall topography during the reactivation of spatial and verbal memory representations became manifest as significant interactions of experiment $\times$ electrode position in both experimental sections [in the learning phase, $F(17,442)=11.17, p<.01$; in the retrieval phase, $F(17,442)=17.35, p<.01]$. Finally, electrodespecific comparisons calculated for the factor of level of specific concepts revealed a reliable two-way interaction of experiment $\times$ fan for the electrode locations $\mathrm{F} 3[F(2,26)$ $=4.22]$ and $\mathrm{Pz}[F(2,26)=6.38]$ (both $p s<.05)$.

\section{GENERAL DISCUSSION}

Two experiments were performed in order to test whether cortical activation patterns have the same or a different topography when distinct kinds of information are reactivated in long-term memory. Both experiments were completely equivalent with respect to the following characteristics: the number of items and the number of interitem associations that had to be learned, the learning and the overlearning procedure, the criterion that had to be passed before the retrieval test was started, and the retrieval test itself. Moreover, in both experiments, slow ERPs as indicators of cortical activation were recorded in only the time epochs where the same stimuli were presented to the subjects. The only difference between the two experiments concerned the type of information that had to be reactivated in long-term memory (i.e., spatial mediators in Experiment 1 and verbal mediators in Experiment 2). Last, but not least, a paradigm was used that invoked genuine memory recall and in which the subject had no other options than to solve the task by retrieving the learned associations between concepts and mediators (see Heil et al., 1994).

In the two experiments, three conditions were created that, according to the RTs, imposed equivalent but systematically increasing processing demands on the subject. By using different cues and mediators in the fan paradigm, it was possible to reactivate one, two, or three 


\section{Verbal Information}

\section{Anticipation}
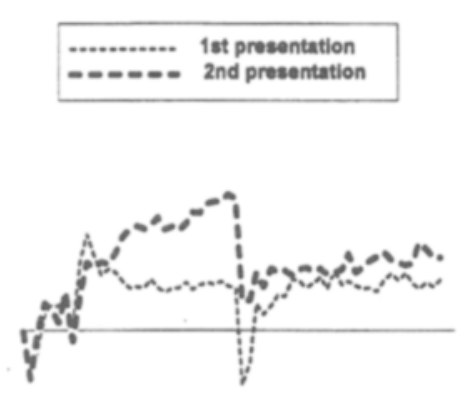

F3

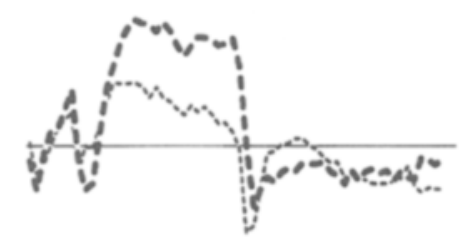

$\mathrm{Pz}$

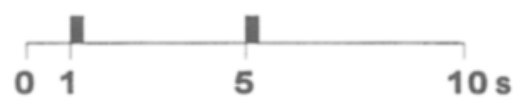

\section{Retrieval}

specific probes

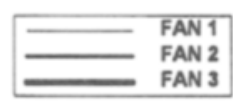

Figure 11. Summary of the main results of Experiment 2 for electrode positions F3 and Pz.

representations within distinct code domains: spatial representations were triggered by the specific concepts of Experiment 1, verbal representations by the specific concepts of Experiment 2, and pictorial representations by the general concepts of both experiments. Within the first two code domains (spatial and verbal), experimentally trained, episodic associations had to be reactivated, whereas, in the third code domain, a priori given semantic knowledge had to be accessed. Irrespective of these differences in the quality of the reactivated information, all three manipulations resulted in a comparable increase of RT with an increasing number of accessed representations. This replicates the results of Heil et al . (1994) and suggests once more that the temporal dynamics of reactivation processes in long-term memory do not differ in principle for verbal, spatial, and pictorial information. Although this finding is compatible with the idea of an abstract propositional code for all three types of information, it is also compatible with the idea of separate neural networks specialized for processing and storage of distinct kinds of information (see, e.g., Hinton et al., 1986). However, the statement that distinct but equivalently organized cell assemblies exist that are specialized for material-specific representations must remain empty as long as there is no converging evidence for it from empirical sources other than RTs. Strong proof would be if such distinct assemblies could be localized within dis- tinct parts of the human brain. Slow ERPs provided the method to test this hypothesis in a very specific manner. On the basis of effects observed with brain-lesioned patients, precise predictions are possible about where in the cortex such specialized modules could be located and where the most prominent neuronal activation should be found in an intact brain during retrieval of different memory contents: The retrieval of spatial information should result in an activation of the parietal cortex, and the retrieval of verbal information should result in an activation of the left frontal cortex, respectively.

In the present study, these general predictions were not tested only in that the overall topography of slow waves was compared when the subjects had to reactivate either verbal or nonverbal memory contents. Rather, there were eight hypotheses relating specific and well-defined experimental manipulations to particular slow-wave effects. First, the activation of spatial memory contents by means of the anticipation method in the learning phase should result in an activation of the parietal cortex. Second, this parietal activation should also be present when the same spatial material is accessed again in an explicit retrieval situation. Third, the activation of the parietal cortex should be higher the more spatial information had to be reactivated (fan manipulation for specific concepts). Fourth, for general concepts, the fan manipulation should not result in different amplitudes over the parietal cor- 
tex. In principle, the very same four predictions should hold, if verbal information had to be retrieved from longterm memory, but all effects should then be found over the left frontal cortex. As a matter of fact, all of these predictions could be substantiated.

\section{Topography of Activation Patterns and the Location of Memory Reactivation Processes}

Our results suggest that distinct neural generators are involved when spatial and verbal information is activated in memory. This follows directly from the distinct topography of the negative slow waves observed in the two experiments. Still open, however, is the question of where these generators are located. As a matter of fact, one and the same set of electrical (or magnetic) measurements registered from the surface of the scalp can, in principle, be generated by an infinite number of arrangements of neural generators inside of the head (Nunez, 1981). Therefore, statements about the localization of activation foci within the brain are ambiguous unless there are other sources of information that restrict the degrees of freedom. ${ }^{2}$ As far as slow ERPs are concerned, several findings support the hypothesis that these are, in fact, generated in cortical areas next to the recording site (for a summary, see, e.g., Birbaumer, Elbert, Canavan, \& Ruckstroh, 1990). The following pieces of evidence all converge to this conclusion:

1. Parallel recordings of slow waves and intracranial electrical activity revealed a close relationship between surface negative potentials and a predominance of EPSPs in the upper layers of the cortex (Mitzdorf, 1991; Speckmann et al., 1984).

2. When slow ERPs and regional cerebral blood-flow measures are recorded in the same experimental paradigm, a close correspondence can be observed between the topography of foci of energy consumption in the cortex and the topography of slow waves over the scalp (M. Lang et al., 1988).

3. Model calculations with realistic head models reveal that slow potentials, as observed in the present study, cannot originate from deep structures because of the more random orientation of polarized layers within subcortical brain structures and because of the substantial attenuation of volume-conducted currents that originate in deeper structures (Braun et al., 1990).

Therefore, it seems justifiable to assume that slow waves in the EEG, as observed in the present study, have a cortical generator in close proximity to the recording site. Thus, the data support the conclusion that retrieval of spatial and verbal information from episodic longterm memory is closely linked to an activation of distinct cell assemblies located in the parietal and in the left frontal cortex, respectively.

\section{Dissociation of Physiological and Behavioral Measurements}

In the two experiments, the effects observed with the ERP and the RT measurements are not completely equivalent. With general concepts used as probes, semantic knowledge about types and exemplars of object categories had to be activated, and, on the basis of this knowledge, the relevant specific concepts could be generated (see Figure 1). As far as the present set of data is concerned, these processes led to a significant and systematic increase of RT, but they left no signature in the slow waves, neither in form of a specific topography nor in form of an amplitude change. Specific concepts, on the other hand, that directly induced an activation of experimentally established associations led to an increase in RT and to an increase in the slow-wave amplitude when more associations had to be scanned. According to Tulving's (1991) conceptual distinction between semantic and episodic memory, the links between specific concepts and mediators have the quality of episodic representations, and the links between the categories and the exemplars have the quality of semantic representations, respectively. This would imply that the slow-wave effects observed in our two experiments were due primarily to reactivation processes of episodic representations. Of course, this rather specific functional interpretation of the slow-wave effects is post hoc. In fact, in our experiments, the proposed episodic-semantic distinction between specific and general concepts is confounded with the quality of the to-be-retrieved information. The supposed episodic links refer to spatial or verbal information, whereas the supposed semantic links refer to pictorial information. According to the localized cortical cell assembly approach, the retrieval of episodic pictorial information should result in an activation of the temporal cortex. Obviously, further experimental evidence is needed to answer this question.

However, the different pattern of results, as such, that was found for specific and general probes demonstrates that effects that can be observed by means of slow eventrelated EEG potentials are not redundant to RTs or error rates, nor are behavioral data redundant to the psychophysiological ones.

Finally, one might wonder why the temporal dynamics of reactivation of different types of information should be so highly similar. Though different parts of the cortex are responsible for spatial versus verbal information, they might be doing roughly the same things on rather different contents (i.e., reactivating memory representations). In our experimental paradigm, not much processing of these kinds of information is needed, whose temporal dynamics probably would differ for spatial versus verbal information. Recently, we (Heil, Rauch, \& Rösler, 1995) combined the retrieval of spatial versus verbal information within a modified fan paradigm with no secondary task, a mental rotation task, or a syntactic lexical decision task. In the control condition, RT functions again did not differ for spatial and verbal information. In contrast, a more pronounced fan effect for spatial information was found with mental rotation as secondary task, whereas the reversed pattern of results was obtained with syntactic lexical decision as secondary task. These results not only validate the topographical dissociation obtained by means of slow potentials in the EEG but they 
show that behavioral data can do more than just measure "overall effort."

\section{Conclusion}

The findings of the two experiments are compatible with an information-processing model that assumes materialspecific cell assemblies in which episodic memory contents are represented and processed (Damasio, 1989; Hinton et al., 1986). Of course, our results do not falsify -in the strict epistemological sense-theories that plead for the existence of a common, abstract representation somewhere in the brain (Anderson, 1978). It is still possible that an abstract code exists and that it is downloaded into cortical cell assemblies that do not store anything but that are specialized only for higher order processing of phenomenologically distinct memory contents (Kosslyn, 1991). However, quite arbitrary post hoc assumptions would have to be introduced if such commoncode theories are to be brought into account with the present set of data. For the time being, neuroscience has not provided much compelling evidence for the existence of a common, abstract representation somewhere in the brain (see Damasio, 1989). Although lesion studies suggest only that temporal lobe structures are very likely a prerequisite for solving a wide variety of distinct memory tests (Markowitsch, 1984; Squire, 1992), the same data also show that the functional role of the temporal lobe is not equivalent to that postulated for a master store. Most likely, this brain region controls whether or not an item is stored at all and participates in processes that guarantee access to representations that are stored elsewhere in distributed cell assemblies (McClelland, McNaughton, \& O'Reilly, 1995). Since slow waves, as recorded in the present study, do not systematically reflect the activation level of cell assemblies that are located deeper in the brain, our results cannot tell anything about the functional role of subcortical structures during long-term memory retrieval. These structures could or could not be activated in our task; we cannot tell. To settle this question, other brain imaging techniques, such as PET or fMRI, have to be used, and the results obtained with these measurements have to be related to the ERP findings and the RT functions.

\section{REFERENCES}

ANDERSON, J. R. (1974). Retrieval of propositional information from long-term memory. Cognitive Psychology, 6, 451-474.

ANDERSON, J. R. (1976). Language, memory, and thought. Hillsdale, NJ: Erlbaum.

ANDERSON, J. R. (1978). Arguments concerning representations for mental imagery. Psychological Review, 85, 249-277.

ANDERson, J. R., \& PAulson, R. (1978). Interference in memory for pictorial information. Cognitive Psychology, 10, 178-202.

Birbaumer, N., Elbert, T., Canavan, A. G. M., \& Rockstroh, B. (1990). Slow potentials of the cerebral cortex and behavior. Physiological Reviews, 70, 1-41.

Braitenberg, V., \& Schütz, A. (1991). Anatomy of the cortex. Heidelberg: Springer-Verlag.

Braun, C., Lutzenberger, W., Miltner, W., \& Elbert, T. (1990) Can subcortical structures generate potentials large in amplitude? In
C. H. M. Brunia, A. W. K. Gaillard, \& A. Kok (Eds.), Physiological brain research (pp. 31-35). Tilburg: Tilburg University Press.

BRUNia, C. H. (1988). Movement and stimulus preceding negativity. Biological Psychology, 26, 165-178.

BRUNia, C. H., \& DAMEN, E. J. (1988). Distribution of slow brain potentials related to motor preparation and stimulus anticipation in a time estimation task. Electroencephalography \& Clinical Neurophysiology, 69, 234-243.

Creutzfeld, O. D. (1983). Cortex Cerebri: Leistung, strukturelle und funktionelle Organisation der Hirnrinde [Cerebral cortex: Power, structural and functional organization of the cortex]. Berlin: SpringerVerlag.

Damasio, A. R. (1989). Time-locked multiregional retroactivation: A system-level proposal for the neural substrates of recall and recognition. Cognition, 33, 25-62.

DE RENZi, E. (1982). Memory disorders following focal neocortical damage. Philosophical Transactions of the Royal Society of London. Series $B, 298,73-83$.

Ehrlichman, H., \& Barrett, J. (1983). Right hemispheric specialization for mental imagery: A review of the evidence. Brain \& Cognition, 2, 55-76.

ElberT, T., \& RockstroH, B. (1987). Threshold regulation-A key to the understanding of the combined dynamics of EEG and eventrelated potentials. Journal of Psychophysiology, 4, 317-333.

FraCKOWIAK, R. S. J. (1994). Functional mapping of verbal memory and language. Trends in Neurosciences, 17, 109-115.

Georgopoulos, A. P., Lurito, J. T., Petrides, M., Schwartz, A. B. \& MASSEY, J. T. (1989). Mental rotation of the neuronal population vector. Science, 243, 234-236.

HeIL, M., RAUCH, M., \& RösLER, F. (1995). Double-dissociating retrieval of spatial and verbal information from episodic long-term memory by means of a dual-task approach. Manuscript submitted for publication.

Heil, M., Rösler, F., \& Hennighausen, E. (1994). Dynamics of activation in long-term memory: The retrieval of verbal, pictorial, spatial, and color information. Journal of Experimental Psychology: Learning, Memory, \& Cognition, 20, 185-200.

Hennighausen, E., Heil, M., \& Rösler, F. (1993). A correction method for DC-drift artifacts. Electroencephalography \& Clinical Neurophysiology, 86, 199-204.

Hinton, G. E., MCClelland, J. L., \& Rumelhart, D. E. (1986). Distributed representations. In D. E. Rumelhart, J. L. McClelland, \& the PDP Research Group (Eds.), Parallel distributed processing: Vol. 1. Foundations (pp. 77-109). Cambridge, MA: MIT Press.

Horwitz, B., Grady, C. L., HaXby, J. V., Ungerleider, L. G., Schapiro, M. B., MISHKIN, M., \& RAPOPORT, S. I. (1992). Functional associations among human posterior extrastriate brain regions during object and spatial vision. Journal of Cognitive Neuroscience, 4, 31 1-322.

HUYNH, H., \& FELDT, L. S. (1976). Estimation of the box correction for degrees of freedom from sample data in randomized block and splitplot designs. Journal of Eductaion Statistics, 1, 69-82.

Jetter, W., Poser, U., Freeman, R. B., \& Markowitsch, H. J. (1986). A verbal long-term memory deficit in frontal lobe damaged patients. Cortex, 22, 229-242.

Kahnemann, D. (1973). Attention and effort. Englewood, NJ: PrenticeHall.

KöHLER, S., Kapur, S., Moscovitsch, M., \& Houle, S. (1995). Brain regions activated during retrieval of spatial location and object identity information from long-term memory: A positron emission tomography study. Manuscript submitted for publication.

KossLYN, S. M. (1987). Seeing and imagining in the cerebral hemispheres: A computational approach. Psychological Review, 94, $148-175$

KosSLYN, S. M. (1991). A cognitive neuroscience of visual cognition: Further developments. In R. H. Logie \& M. Denis (Eds.), Mental images in human cognition (pp. 351-381). Amsterdam: Elsevier. Kossly , S. M. (1994). Image and brain. Cambridge: MIT Press.

Kosslyn, S. M., Holtzman, J. D., Gazzaniga, M. S., \& Farah, M. J. (1985). A computational analysis of mental image generation: Evidence from functional dissociations in split-brain patients. Journal of Experimental Psychology: General, 114, 311-341. 
Lang, M., Lang, W., Podreka, I., Steiner, M., Uhl, F., Suess, E. MÜLLER, C., \& DEECKE, L. (1988). DC-potential shifts and regional cerebral blood flow reveal frontal cortex involvement in human visuomotor learning. Experimental Brain Research, 71, 353-364.

Lang, W., Zilch, O., Koska, C., Lindinger, G., \& Deecke, L. (1989). Negative cortical DC shifts preceding and accompanying simple and complex sequential movements. Experimental Brain Research, 74, 99-104.

LeVINE, D. N., Warach, J., \& Farah, M. J. (1985). Two visual systems in mental imagery: Dissociation of "what" and "where" in imagery disorders due to bilateral posterior cerebral lesions. Neurology, 35 , $1010 \cdot 1018$

MaRKOWITSCH, H. J. (1984). Can amnesia be caused by damage of a single brain structure? Coriex, 20, 27-45.

McCAllum, W. C., \& CurRy, S. H. (EDs.) (1993). Slow potential changes in the human brain. New York: Plenum.

MCCARThY, G., \& WoOD, C. C. (1985). Scalp distributions of eventrelated potentials: An ambiguity associated with analysis of variance models. Electroencephalography \& Clinical Neurophysiology, 62 . 203-208.

McCarthy, R. A., \& Warrington, E. K. (1990). Cognitive neuropsychology. San Diego, CA: Academic Press.

McClelland, J. L., McNaughton, B. L., \& O'Reilly, R. C. (1995) Why there are complementary learning systems in the hippocampus and neocortex: Insights from the successes and failures of connectionist models of learning and memory. Psychological Review, 102 , 419-457.

Mishkin, M., UnGerleider, L. G., \& Macko, K. A. (1983). Object vision and spatial vision: Two cortical pathways. Trends in Neurosciences, 6, 414-417.

Mitzdorf, U. (1991). Physiological sources of evoked potentials. In C. H. M. Brunia, G. Mulder, \& M. N. Verbaten (Eds.), Event-related brain research (pp. 47-57). Amsterdam: Elsevier.

NADEL, L. (1992). Multiple memory systems: What and why. Journal of Cognitive Neuroscience, 4, 179-188.

NUNEZ, P. (1981). Electrical fields of the brain: The neurophysics of $E E G$. New York: Ox ford University Press.

Paivio, A. (1986). Mental representations. New York: Oxford University Press.

Peterson, S. E., Fox, P. T., Posner, M. I., Mintun, M., \& Raichle, M. E. (1988). Positron emission tomographic studies of the cortical anatomy of single-word processing. Nature, 331, 585-589.

RAICHLE, M. E. (1994). Images of the mind: Studies with modern imaging techniques. Annual Review of Psychology, 45, 333-356.

Risse, G. L., Rubens, A. B., \& Jordan, L. S. (1984). Disturbances of long-term memory in aphasic patients: A comparison of anterior and posterior lesions. Brain, 107, 605-617.

RösLER, F., \& HEIL, M. (1991). Towards a functional categorization of slow waves: Taking into account past and future events. Psychophysiology, 28, 344-358.

Rösler, F., Heil, M., Bajrić, J., Pauls, A. C., \& Hennighausen, E. (1995). Patterns of cerebral activation while mental images are rotated and changed in size. Psychophysiology, 32, 135-149.

RöSleR, F., HeIL, M., \& Glowalla, U. (1993). Monitoring retrieval from long-term memory by slow event-related brain potentials. Psychophysiology, 30, 170-182.

Rösler, F., HeIL, M., \& Hennighausen, E. (1995). Distinct cortical ac- tivation patterns during long-term memory retrieval of verbal, spatial, and color information. Journal of Cognitive Neuroscience, 7, 53-67. Rösler, F., Röder, B., Heil., M., \& Hennighausen, E. (1993). Topographic differences of slow event-related brain potentials in blind and sighted adult human subjects during haptic mental rotation. Cognitive Brain Research, 1, 145-159.

RugG, M. D., \& Doyle, M. C. (1994). Event-related potentials and stimulus repetition in direct and indirect tests of memory. In H.-J. Heinze, T. F. Münte, \& G. R. Mangun (Eds.), Cognitive electrophysiology (pp. 124-148). Boston: Birkhäuser.

Speckmann, E. J., Caspers, H., \& Elger, C. (1984). Neuronal mechanisms underlying the generation of field potentials. In T. Elbert, B. Rockstroh, W. Lutzenberger, \& N. Birbaumer (Eds.), Self-regulation of the brain and behavior (pp. 9-25). Heidelberg: Springer-Verlag.

SQUiRE, L. R. (1992). Memory and hippocampus: A synthesis from findings with rats, monkeys, and humans. Psychological Review, 99, 195-231.

STOPHER, K., \& KirSner, K. (1981). Long-term memory for pictures and sentences. Memory \& Cognition, 9, 34-40.

Tulving, E. (1991). Concepts of human memory. In L. R. Squire, N. M. Weinberger, G. Lynch, \& J. L. McGaugh (Eds.), Memory: Organization and locus of change (pp. 3-34). New York: Oxford University Press.

Uhl, F., GoldenberG, G., LanG, W., Lindinger, G., Steiner, M., \& DEECKE, L. (1990). Cerebral correlates of imaging colours, faces and a map: II. Negative cortical DC potentials. Neuropsychologia, 28, 81-93.

VON DER MALSBURG, C. (1981). The correlation theory of brain function. Göttingen, Germany: Max-Planck Institute for Biophysical Chemistry, Department of Neurobiology.

WICKENS, C. D. (1984). Engineering psychology and human performance. Columbus, $\mathrm{OH}$ : Merrill.

\section{NOTES}

1. In addition to the slow negative ERP effects mentioned, the repetition of the drawings was accompanied by a widespread positive peak at about $600 \mathrm{msec}$. This positivity resolves into a negativity a few hundred milliseconds later. The component seems to be equivalent to the repetition positivity described, for example, by Rugg and Doyle (1994). This positivity, which was less distinct in Experiment 2, was not the topic of our study, and, therefore, results concerning this component will not be reported.

2. This indeterminancy is not unique for EEG or MEG data; however, the situation is similar-although because of other reasons-for other brain imaging techniques. With EEG and MEG measures, the indeterminancy concerns, in particular, the location of a generator within the three-dimensional space of the head. With PET, the relationship between an increase in neural activity and its metabolic consequences is not totally understood, and indeterminancies exist with respect to the exact spatial location of an activation focus within a larger volume segment showing heightened activity and with respect to the exact temporal relationship between electrical and metabolic activity. Morover, an increase in metabolic activity must not necessarily be due to an increase of excitatory activity in the nervous tissue; it could also be due to an increase of inhibitory activity.

(Manuscript received May 8, 1995; revision accepted for publication December 12, 1995.) 